\title{
Dynamic boundary conditions as a singular limit of parabolic problems with terms concentrating at the boundary
}

\author{
Ángela Jiménez-Casas and Aníbal Rodríguez-Bernal \\ Communicated by James Robinson, received October 25, 2011.
}

\begin{abstract}
We obtain nonhomogeneous dynamic boundary conditions as a singular limit of a parabolic problem with null flux and potentials and reaction terms concentrating at the boundary.
\end{abstract}

\section{Contents}

1. Introduction 341

2. On the well-posedness of the approximating and limit problems 344

3. Concentrating integrals $\quad 349$

$\begin{array}{ll}\text { 4. Singular limit as } \varepsilon \rightarrow 0 & 359\end{array}$

$\begin{array}{ll}\text { References } & 367\end{array}$

\section{Introduction}

Dynamic boundary conditions have the main characteristic of involving the time derivative of the unknown. They have been used, among others, as a model of "boundary feedback" in stabilization and control problems of membranes and plates, $\mathbf{3}, \mathbf{1 3}, \mathbf{1 4}, \mathbf{1 2}, \mathbf{1 5}, \mathbf{2 3}$, in phase transition problems, $\mathbf{2 2}, \mathbf{7}, \mathbf{8}, \mathbf{9}, \mathbf{1 7}, \mathbf{4}$, in some hydrodynamic problems, 10, 21 or in population dynamics, 6]. They have also been considered in the context of elliptic-parabolic problems, [5, 18. Also several of so called "transmission problems" have been described and analyzed in 20], some of which lead, under some singular perturbation limits, to problems with dynamical boundary conditions.

1991 Mathematics Subject Classification. 35.

Key words and phrases. Dynamic boundary conditions, parabolic problems, concentrating integrals, singular perturbation.

Partially suppported by Projects MTM2009-07540, GR58/08 Grupo 920894 BSCH-UCM, Grupo de Investigación CADEDIF and FIS2009-12964-C05-03, Spain. 


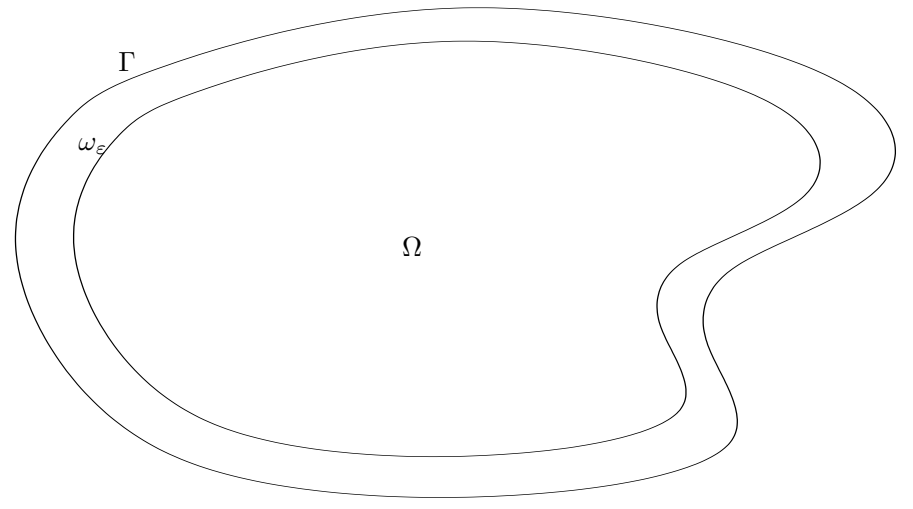

Figure 1. The set $\omega_{\varepsilon}$

In this paper our goal is to prove that dynamic boundary conditions can be obtained as the singular limit of elliptic/parabolic problems in which the time derivative concentrates in a narrow region close to the boundary.

To be more precise, let $\Omega$ be an open bounded smooth set in $\mathbb{R}^{N}$ with a $C^{2}$ boundary $\Gamma=\partial \Omega$. Define the strip of width $\varepsilon$ and base $\Gamma$ as

$$
\omega_{\varepsilon}=\{x-\sigma \vec{n}(x), x \in \Gamma, \sigma \in[0, \varepsilon)\}
$$

for sufficiently small $\varepsilon$, say $0 \leq \varepsilon \leq \varepsilon_{0}$, where $\vec{n}(x)$ denotes the outward normal vector. We note that the set $\omega_{\varepsilon}$ is a neighborhood of $\Gamma$ in $\bar{\Omega}$, that collapses to the boundary when the parameter $\varepsilon$ goes to zero.

Then we consider the following family of parabolic problems

$$
\left\{\begin{aligned}
\frac{1}{\varepsilon} \mathcal{X}_{\omega_{\varepsilon}} u_{t}^{\varepsilon}-\Delta u^{\varepsilon}+\lambda u^{\varepsilon}+\frac{1}{\varepsilon} \mathcal{X}_{\omega_{\varepsilon}} V_{\varepsilon}(x) u^{\varepsilon} & =f+\frac{1}{\varepsilon} \mathcal{X}_{\omega_{\varepsilon}} g_{\varepsilon} & & \text { in } \Omega \\
\frac{\partial u^{\varepsilon}}{\partial n} & =0 & & \text { on } \Gamma \\
u^{\varepsilon}(0, x) & =u_{0}^{\varepsilon}(x) & & \text { in } \Omega
\end{aligned}\right.
$$

where $\mathcal{X}_{\omega_{\varepsilon}}$ is the characteristic function of the set $\omega_{\varepsilon}$ and $\lambda \in \mathbb{R}$.

As $\omega_{\varepsilon}$ shrinks to the boundary as $\varepsilon \rightarrow 0$, the goal in this work is to show that dynamic boundary conditions can be obtained as a result of this limiting process. More precisely, the main result in this work is to prove that the family of solutions, $u^{\varepsilon}$, converges in some sense, when the parameter $\varepsilon$ goes to zero, to a limit function $u^{0}$, which is given by the solution of the following parabolic problem with dynamic boundary conditions

$$
\left\{\begin{aligned}
-\Delta u^{0}+\lambda u^{0} & =f & & \text { in } \Omega \\
u_{t}^{0}+\frac{\partial u^{0}}{\partial n}+V(x) u^{0} & =g & & \text { on } \Gamma \\
u^{0}(0, x) & =v_{0}(x) & & \text { on } \Gamma
\end{aligned}\right.
$$

where $v_{0}, V$ and $g$ are obtained as the limits of the concentrating terms

$$
\frac{1}{\varepsilon} \mathcal{X}_{\omega_{\varepsilon}} u_{0}^{\varepsilon} \rightarrow v_{0}, \quad \frac{1}{\varepsilon} \mathcal{X}_{\omega_{\varepsilon}} V_{\varepsilon} \rightarrow V, \quad \frac{1}{\varepsilon} \mathcal{X}_{\omega_{\varepsilon}} g_{\varepsilon} \rightarrow g
$$

in some sense that we make precise below. In particular, we will obtain that the time derivative of the solution concentrates to the time derivative of the restriction to the boundary, as $\varepsilon \rightarrow 0$. Notice that all concentrating terms in (1.1) are transferred, in the limit, to the boundary condition in (1.2). 
Related problems have been considered before. Paper [2] considered linear elliptic problems with concentrating terms near the boundary. Also 11] considered nonlinear parabolic problems with linear and nonlinear terms concentrating near the boundary and analyzed the proximity of the long time behavior of solutions by studying the proximity of the the corresponding global attractors. In both [1] and in this paper the results in 2 provide some of the building blocks of the analysis. Note however that the case considered here is more singular than the ones in the references quoted above, because the singular limit affects the time derivative of the solution. The reader is also referred to [20, 1] and references therein for some other cases in which similar singular limits arise.

As noted in 2, in the context of elliptic problems, the convergence results obtained below, despite its intrinsic mathematical interest, have potential applications in developing approximation schemes for (1.2). Numerical solutions of (1.1) can be obtained by suitable spectral or finite element methods. In both cases the setting gets rid of the zero flux condition. In fact, (1.1) has a natural and simple variational formulation not involving surface integrals or traces in $\Gamma$. On the other hand, solving (1.2) requires to use suitable sets of functions defined on the boundary, whose trace evolves according to the second equation in (1.2).

This approach becomes more subtle if the boundary of the domain is not smooth enough. In fact if the domain is not smooth, it is troublesome to give a meaning to the boundary condition in (1.2), although (1.1) has a natural and simple variational formulation not involving surface integrals or traces. Hence the limit functions of (1.1) can be taken as proper way of defining solutions of (1.2).

Note that (1.1) is formally equivalent to solving

$$
\left\{\begin{aligned}
-\Delta u^{\varepsilon}+\lambda u^{\varepsilon} & =f & & \text { in } \Omega \backslash \bar{\omega}_{\varepsilon} \\
\frac{1}{\varepsilon} u_{t}^{\varepsilon}-\Delta u^{\varepsilon}+\lambda u^{\varepsilon}+\frac{1}{\varepsilon} V_{\varepsilon} u^{\varepsilon} & =f+\frac{1}{\varepsilon} g_{\varepsilon} & & \text { in } \omega_{\varepsilon} \\
\frac{\partial u^{\varepsilon}}{\partial n} & =0 & & \text { on } \Gamma \\
u^{\varepsilon}(0, x) & =u_{0}^{\varepsilon}(x) & & \text { in } \Omega
\end{aligned}\right.
$$

and that in (1.4) boundary conditions are missing on $\Gamma_{\varepsilon}=\partial \omega_{\varepsilon} \backslash \Gamma=\partial\left(\Omega \backslash \bar{\omega}_{\varepsilon}\right)$. Since there would be several ways of connecting the solutions of the elliptic and the parabolic equations in (1.4) along that boundary, we consider the boundary conditions on $\Gamma_{\varepsilon}$ that ensure maximal smoothness of solutions. This is achieved by imposing the classical transmissions conditions on $\Gamma_{\varepsilon}$, that is, no jump of the $u^{\varepsilon}$ and its normal derivate across $\Gamma_{\varepsilon}$, see $\mathbf{1 9}$,

$$
\left[u_{\varepsilon}\right]_{\Gamma_{\varepsilon}}=\left[\frac{\partial u_{\varepsilon}}{\partial n}\right]_{\Gamma_{\varepsilon}}=0
$$

Hence, (1.4) and (1.5) is a formulation of an elliptic-parabolic transmission problem, see 16, Chapter 1, Section 9, for related problems. The well-posedness of (1.1), in the sense of (1.4), (1.5), will be addressed in Section 2.1 following the techniques in 19 .

On the other hand, (1.2) must be understood as an evolution problem on the boundary $\Gamma$, such that, for each time $t>0$, the solution must be lifted to the interior of $\Omega$ by means of the elliptic equation in (1.2). In this way the term $\frac{\partial u^{0}}{\partial n}$, which is the so called Dirichlet Neumann operator, becomes a linear nonlocal operator for functions defined on $\Gamma$. The well-posedness of (1.2) will be discussed in Section 2.2 following the techniques in $\mathbf{1 8}$. 
Concerning (1.3) the starting point are the results in 2 which state that if we consider a family of functions in $\Omega$ such that for some $p>1$

$$
\frac{1}{\varepsilon} \int_{\omega_{\varepsilon}}\left|h_{\varepsilon}\right|^{p} \leq C
$$

then, taking subsequences if necessary, one can assume that there exists $h_{0} \in L^{p}(\Gamma)$ such that for any smooth function $\varphi$, defined in $\bar{\Omega}$, we have

$$
\lim _{\varepsilon \rightarrow 0} \frac{1}{\varepsilon} \int_{\omega_{\varepsilon}} h_{\varepsilon} \varphi=\int_{\Gamma} h_{0} \varphi
$$

In other words, the results above indicate that concentrating integrals near the boundary behave as boundary integrals and the concentrating functions behave as traces. Several results of this type for functions that also depend on time, will be obtained in Section 3

These results will be used then in Section 4 when proving that actually solutions of (1.1) converge to solutions of (1.2); see Theorem 4.1 and Theorem 4.3 which are the two main results concerning convergence of solutions. It is worth noting that we will not assume the linear potentials $V_{\varepsilon}$ are nonnegative nor uniformly bounded, but we will rather require the uniform integrability condition above for $p=\rho>N-1$. In fact, for $\varepsilon>0$ fixed, only $\rho>N / 2$ is required in (1.1) for the elliptic part of the equation to be well defined. However for dealing with that family of problems, uniformly in $\varepsilon$, we need $\rho>N-1$, since in the limit the interior potential behaves as a boundary potential which requires this sort of integrability. Indeed for part of the stronger convergence result in Theorem 4.3 we will actually require $\rho>2(N-1)$. Although this may seem a technical restriction, we have fought unsuccesfuly to remove it.

Acknowledgments The author would like to thank several comments from the anonymous referee which greatly helped in improving several points in the paper.

\section{On the well-posedness of the approximating and limit problems}

In this section we describe the well-posedness results for (1.4) and (1.2). For this we will make use of minor variations of the results in $[\mathbf{1 8}, \mathbf{1 9}$.

Here and below $H^{s}(\Omega)$ denote, for $s \geq 0$, the standard Sobolev spaces and for $s>0$ we denote

$$
H^{-s}(\Omega)=\left(H^{s}(\Omega)\right)^{\prime} .
$$

Also $H_{0}^{-1}(\Omega)$ will denote the dual space of $H_{0}^{1}(\Omega)$. Finally, we will consider below traces on $\Gamma$ of functions defined in $\Omega$. Hence, we will denote either by $\gamma(u)$ or by $u_{\mid \Gamma}$ the trace of a function $u$. As above, $H^{-1 / 2}(\Gamma)$ will denote the dual space of $H^{1 / 2}(\Gamma)$.

2.1. Well-posedness of (1.4). Note that in [19] a very similar problem to (1.4) was considered. In fact in [19] Dirichlet boundary conditions were assumed on $\Gamma$ instead as Neumann ones as in this paper. Also it was assumed $V_{\varepsilon}=0$. Therefore, we explain below how to modify the arguments in $\mathbf{1 9}$ to apply them to (1.4). See Theorem 1.1, Theorem 4.9 and Proposition 4.10 in [19].

Hence, we consider (1.4). Since $\varepsilon>0$ is fixed, and in order to simplify the notation, we do not make explicit the dependence on $\varepsilon$. Also, we first concentrate 
in the time-independent, i.e. elliptic, equation associated with (1.4)

$$
\left\{\begin{aligned}
-\Delta u+\mathcal{X}_{\omega} V u+\lambda u & =h & & \text { in } \Omega \\
\frac{\partial u}{\partial n} & =0 & & \text { on } \Gamma .
\end{aligned}\right.
$$

For this, we identify $L^{2}(\Omega)$ with its dual and denote by $H^{-1}(\Omega)$ the dual space of $H^{1}(\Omega)$ and then $H^{1}(\Omega) \subset L^{2}(\Omega) \subset H^{-1}(\Omega)$. Also, we define the bilinear symmetric form in $H^{1}(\Omega)$

$$
a(\varphi, \phi)=\int_{\Omega} \nabla \varphi \nabla \phi+\int_{\omega} V \varphi \phi+\lambda \int_{\Omega} \varphi \phi
$$

for every $\varphi, \phi \in H^{1}(\Omega)$. Assumed

$$
V \in L^{\rho}(\omega), \quad \rho>N / 2,
$$

this bilinear form defines an linear mapping, $L$, between $H^{1}(\Omega)$ and its dual $H^{-1}(\Omega)$.

Now we show that solving (2.1) reduces naturally to solving some problems in $\omega$ and in $\Omega \backslash \bar{\omega}$. For this, we also identify $L^{2}(\omega)$ with its dual and consider the bilinear form restricted to $H^{1}(\omega)$, that is,

$$
a_{\omega}(\varphi, \phi)=\int_{\omega} \nabla \varphi \nabla \phi+\int_{\omega} V \varphi \phi+\lambda \int_{\omega} \varphi \phi
$$

for every $\varphi, \phi \in H^{1}(\omega)$ and denote by $L_{\omega}$ the corresponding linear mapping between $H^{1}(\omega)$ and $H^{-1}(\omega)$.

Then, we have

Definition 2.1. Denote $\Gamma_{*}=\partial \omega \backslash \Gamma=\partial(\Omega \backslash \bar{\omega})$ and $\lambda_{\Omega \backslash \bar{\omega}}$ the first eignvalue of the Laplacian operator with Dirichlet boundary conditions in $\Omega \backslash \bar{\omega}$.

Finally assume $\lambda>-\lambda_{\Omega \backslash \bar{\omega}}$.

i) For $h \in H^{-1}(\Omega \backslash \bar{\omega})$ we define $D(h) \in H^{1}(\Omega)$ as the weak solution of

$$
\left\{\begin{aligned}
-\Delta v+\lambda v & =h & & \text { in } \Omega \backslash \bar{\omega} \\
v & =0 & & \text { on } \Gamma_{*}
\end{aligned}\right.
$$

extended to $\Omega$ by zero in $\omega$.

ii) For a given function $u \in H^{1}(\omega)$, we define $Z(u) \in H^{1}(\Omega \backslash \bar{\omega})$, as the solution of

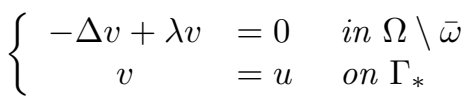

in the sense that

$$
\int_{\Omega \backslash \bar{\omega}} \nabla v \nabla \phi+\lambda \int_{\Omega \backslash \bar{\omega}} v \phi=0
$$

for every $\phi \in H_{0}^{1}(\Omega \backslash \bar{\omega})$ and $v$ satisfies the boundary data on $\Gamma_{*}$.

We also define

$$
B(u)=\left\{\begin{array}{cl}
Z(u) & \text { in } \Omega \backslash \bar{\omega} \\
u & \text { in } \omega .
\end{array}\right.
$$

Therefore, $B$ defines a linear mapping between $H^{1}(\omega)$ and $H^{1}(\Omega)$.

iii) Finally, for functions defined on $\Omega$ we define the "restriction" operator to $\omega$ by $R(u)=\mathcal{X}_{\omega} u$.

With this notations observe that the solution $u \in H^{1}(\Omega)$ of (2.1) must satisfty

$$
u=B\left(\mathcal{X}_{\omega} u\right)+D(h) \text { in } \Omega
$$


so it is determined by $R(u)=\mathcal{X}_{\omega} u \in H^{1}(\omega)$. Now it is easy to see that $R(u)$ which, abusing of the notations we write again $u$, must be a weak solution of the problem in $\omega$

$$
\left\{\begin{aligned}
-\Delta u+V u+\lambda u & =h & & \text { in } \omega \\
\frac{\partial u}{\partial n} & =0 & & \text { on } \Gamma \\
\frac{\partial u}{\partial n_{*}} & =\frac{\partial Z(u)}{\partial n_{*}}+\frac{\partial D(h)}{\partial n_{*}} & & \text { on } \Gamma_{*} .
\end{aligned}\right.
$$

where $n_{*}$ denotes the outward unit normal to $\omega$ along $\Gamma_{*}$. Note that the last boundary condition guarantees the smooth matching across $\Gamma_{*}$, see (1.5).

Finally, the weak solution of (2.3) satisfies

$$
\int_{\omega} \nabla u \nabla v+\int_{\omega} V u v+\lambda \int_{\omega} u v-\int_{\Gamma_{*}} \frac{\partial Z(u)}{\partial n_{*}} v=\int_{\omega} h v+\int_{\Gamma_{*}} \frac{\partial D(h)}{\partial n_{*}} v
$$

for every $v \in H^{1}(\omega)$. This can be written as

$$
A u:=L_{\omega} u-\left(\frac{\partial Z(u)}{\partial n_{*}}\right)_{\Gamma_{*}}=h_{\omega}+\left(\frac{\partial D(h)}{\partial n_{*}}\right)_{\Gamma_{*}} .
$$

Now for the parabolic problem (1.4), observe that solving

$$
\left\{\begin{aligned}
\mathcal{X}_{\omega} u_{t}-\Delta u+\mathcal{X}_{\omega} V u+\lambda u & =h(t) & & \text { in } \Omega, t>0, \\
\frac{\partial u}{\partial n} & =0 & & \text { on } \Gamma \\
u(0, x) & =u_{0}(x) & & \text { in } \Omega,
\end{aligned}\right.
$$

if we assume that for each $t>0$ we have $u(t) \in H^{1}(\Omega)$, we must have

$$
u(t)=B(R(u(t)))+D(h(t)) \quad \text { in } \Omega .
$$

Also the smooth matching across $\Gamma_{*}$, (1.5), now reads

$$
\frac{\partial u}{\partial n_{*}}=\frac{\partial Z(u)}{\partial n_{*}}+\frac{\partial D(h)}{\partial n_{*}} \quad \text { on } \quad \Gamma_{*}, t>0
$$

where $n_{*}$ denotes the outward unit normal to $\omega$ along $\Gamma_{*}$.

Therefore, in view of the properties of the operator $A$ in (2.4), to solve (2.5) we are led to solve an evolution problem of the form

$$
\begin{cases}u(t)=B(v(t))+D(h(t)) & \text { in } \Omega, t>0 \\ v_{t}+A v=h_{\omega}+\left(\frac{\partial D(h)}{\partial n_{*}}\right)_{\Gamma_{*}} & \text { in } \omega, t>0 \\ v(0)=v_{0} & \text { in } \omega\end{cases}
$$

with $A=L_{\omega}-\left(\frac{\partial Z}{\partial n_{*}}\right)_{\Gamma_{*}}$ and assuming that $h(t) \in L^{2}(\Omega)$. Note that in (2.7) we have reduced (2.5) and (2.6) to a nonhomogeneous evolution problem in $\omega$ with a well behaved operator $A$. Finally, note that for (1.4) we take $h=f+\mathcal{X}_{\omega} g$.

Then in a similar fashion as in Theorems 1.1 and 4.9 in [19, we have the following result that states the well-posedness of (2.5), (2.7).

Theorem 2.2. Assume $\lambda>-\lambda_{\Omega \backslash \bar{\omega}}, h \in L^{2}\left((0, T), L^{2}(\Omega)\right)$ and $u_{0} \in L^{2}(\omega)$. i) Then there exists a unique solution of 2.7), which satisfies

$$
u \in C\left([0, T), L^{2}(\omega)\right) \cap L^{2}\left((0, T), H^{1}(\Omega)\right), \quad u(0)=u_{0} \text { in } \omega
$$

and satisfies 2.5) in the sense that

$$
\mathcal{X}_{\omega} u_{t}+L(u)=h \quad \text { in } \quad H^{-1}(\Omega), \quad \text { a.e. } t \in(0, T) .
$$

ii) Assume moreover that either

a) $h \in W^{1,1}\left((0, T), L^{2}(\Omega)\right)$ or 
b) $h \in L^{2}\left((0, T), L^{2}(\omega)\right)=L^{2}((0, T) \times \omega)$ and $h \in W^{1,1}\left((0, T), L^{2}(\Omega \backslash \bar{\omega})\right)$ and $u_{0} \in H^{1}(\Omega)$ satisfies

$$
-\Delta u_{0}+\lambda u_{0}=h(0) \quad \text { in } \Omega \backslash \bar{\omega} .
$$

Then

$$
u \in C\left([0, T), H^{1}(\Omega)\right) \cap L^{2}\left((0, T), H^{2}(\Omega)\right) \quad u(0)=u_{0} \text { in } \Omega
$$

and $u(t)$ satisfies 2.6) a.e. $t \in(0, T)$.

Also, as in Proposition 4.10 in [19, we get

Proposition 2.3. Assume, as above, that $\lambda>-\lambda_{\Omega \backslash \bar{\omega}}$ and $u_{0} \in H^{1}(\Omega)$ satisfying (2.8) and $h(t) \in L^{2}(\Omega)$ a.e. $t \in(0, T)$, are given.

i) If $h \in W^{1,1}\left((0, T), L^{2}(\Omega)\right)$, then

$$
\begin{aligned}
& \|\nabla u(t)\|_{L^{2}(\Omega)}^{2}+\int_{\omega} V u(t)^{2}+\lambda\|u(t)\|_{L^{2}(\Omega)}^{2}+2 \int_{0}^{t} \int_{\omega} u_{t}^{2} \\
& =\left\|\nabla u_{0}\right\|_{L^{2}(\Omega)}^{2}+\int_{\omega} V u_{0}^{2}+\lambda\left\|u_{0}\right\|_{L^{2}(\Omega)}^{2}+ \\
& +2\left(\int_{\Omega} h(t) u(t)-\int_{\Omega} h(0) u_{0}-\int_{0}^{t} \int_{\Omega} h_{t} u\right) .
\end{aligned}
$$

Therefore, the mapping

$$
\left(u_{0}, h\right) \longmapsto\left(u, u_{t}\right)
$$

is Lipschitz from

$$
H^{1}(\Omega) \times W^{1,1}\left((0, T), L^{2}(\Omega)\right)
$$

into

$$
C\left([0, T], H^{1}(\Omega)\right) \times L^{2}((0, T) \times \omega) .
$$

ii) If $h \in L^{2}((0, T) \times \omega)$ and $h \in W^{1,1}\left((0, T), L^{2}(\Omega \backslash \bar{\omega})\right)$, then

$$
\begin{aligned}
& \|\nabla u(t)\|_{L^{2}(\Omega)}^{2}+\int_{\omega} V u(t)^{2}+\lambda\|u(t)\|_{L^{2}(\Omega)}^{2}+2 \int_{0}^{t} \int_{\omega} u_{t}^{2} \\
& =\left\|\nabla u_{0}\right\|_{L^{2}(\Omega)}^{2}+\int_{\omega} V u_{0}^{2}+\lambda\left\|u_{0}\right\|_{L^{2}(\Omega)}^{2}+ \\
& 2\left(\int_{0}^{t} \int_{\omega} h u_{t}+\int_{\Omega \backslash \bar{\omega}} h(t) u(t)-\int_{\Omega \backslash \bar{\omega}} h(0) u_{0}-\int_{0}^{t} \int_{\Omega \backslash \bar{\omega}} h_{t} u\right) .
\end{aligned}
$$

Therefore, the mapping $\left(u_{0}, h_{\omega}, h_{\Omega \backslash \bar{\omega}}\right) \longmapsto\left(u, u_{t}\right)$ is Lipschitz from $H^{1}(\Omega) \times$ $L^{2}((0, T) \times \omega) \times W^{1,1}\left((0, T), L^{2}(\Omega \backslash \bar{\omega})\right)$ into $C\left([0, T], H^{1}(\Omega)\right) \times L^{2}((0, T) \times \omega)$.

2.2. Well-posedness of (1.2). We consider the parabolic problem (1.2), that is

$$
\left\{\begin{aligned}
-\Delta u^{0}+\lambda u^{0} & =f & & \text { in } \Omega \\
u_{t}^{0}+\frac{\partial u^{0}}{\partial n}+V_{0}(x) u^{0} & =g & & \text { on } \Gamma \\
u^{0}(0, x) & =v_{0}(x) & & \text { on } \Gamma
\end{aligned}\right.
$$

for which we adapt the results in $[\mathbf{1 8}$. Note that the setting for this problem is pretty much in the spirit of the previous section, and therefore, we point out the main differences. The reader is then referred to $\mathbf{1 8}$ for full details.

In this case we define the bilinear symmetric form in $H^{1}(\Omega)$

$$
a_{0}(\varphi, \phi)=\int_{\Omega} \nabla \varphi \nabla \phi+\int_{\Gamma} V_{0} \varphi \phi+\lambda \int_{\Omega} \varphi \phi
$$


for every $\varphi, \phi \in H^{1}(\Omega)$. Assuming

$$
V_{0} \in L^{\rho}(\Gamma), \quad \rho>N-1 .
$$

this bilinear form defines a linear mapping, $L_{0}$, between $H^{1}(\Omega)$ and its dual $H^{-1}(\Omega)$.

Definition 2.4. Denote by $\lambda_{\Omega}$ the first eigenvalue of the Laplace operator in $\Omega$ with Dirichlet boundary conditions and assume $\lambda>-\lambda_{\Omega}$.

i) For $h \in H_{0}^{-1}(\Omega)$ we define $D_{0}(h) \in H^{1}(\Omega)$ as the weak solution of

$$
\left\{\begin{aligned}
-\Delta v+\lambda v & =h & & \text { in } \Omega \\
v & =0 & & \text { on } \Gamma
\end{aligned}\right.
$$

ii) For a given function $u$ defined on $\Gamma$, we define $B_{0}(u) \in H^{1}(\Omega)$, as the weak solution of

$$
\left\{\begin{aligned}
-\Delta v+\lambda v & =0 & & \text { in } \Omega \\
v & =u & & \text { on } \Gamma
\end{aligned}\right.
$$

in the sense that

$$
\int_{\Omega} \nabla v \nabla \phi+\lambda \int_{\Omega} v \phi=0
$$

for every $\phi \in H_{0}^{1}(\Omega)$ and $v$ satisfies the boundary data on $\Gamma$.

With this, solving (2.11) is equivalent to solve

$$
\begin{cases}u^{0}(t)=B_{0}\left(\gamma(u(t))+D_{0}(f(t))\right. & \text { in } \Omega \\ u_{t}^{0}+\frac{\partial B_{0}\left(u^{0}(t)\right)}{\partial n}+V_{0} u^{0}=g-\frac{\partial D_{0}(f(t))}{\partial n} & \text { on } \Gamma, t>0 \\ u^{0}(0)=v_{0} & \text { on } \Gamma\end{cases}
$$

assuming $f(t) \in L^{2}(\Omega)$ for $t>0$. Note that $\frac{\partial B_{0}(u)}{\partial n}$ above is the standard DirchletNeumann operator which is well known to be a positive self-adjoint isomorphism between $H^{1 / 2}(\Gamma)$ and $H^{-1 / 2}(\Gamma)$. Thus we have reduced (2.11) to an evolution problem on $\Gamma$.

Now as in Corollary 3.3 in [18 we have the following result that states the well-posedness of (2.11), (2.12).

Proposition 2.5. Assume $\lambda>-\lambda_{\Omega}, f \in L^{2}\left((0, T), L^{2}(\Omega)\right), g \in L^{2}((0, T) \times \Gamma)$ and $v_{0} \in L^{2}(\Gamma)$ are given.

i) Then there exists a unique solution of (2.12) which satisfies

$$
u^{0} \in L^{2}\left((0, T), H^{1}(\Omega)\right), \quad \gamma\left(u^{0}\right)_{t} \in L^{2}((0, T) \times \Gamma)
$$

and satisfies (2.11) in the sense that

$$
\gamma\left(u^{0}\right)_{t}+L_{0}\left(u^{0}\right)=f_{\Omega}+g_{\Gamma}
$$

as an equality in $H^{-1}(\Omega)$, a.e. $t \in(0, T)$. In particular $\gamma\left(u^{0}\right) \in C\left([0, T], L^{2}(\Gamma)\right)$ and $\gamma\left(u^{0}\right)(0)=v_{0}$.

ii) Moreover, if $f \in C\left([0, T), L^{2}(\Omega)\right)$ and $u_{0} \in H^{1}(\Omega)$ satisfies

$$
-\Delta u_{0}+\lambda u_{0}=f(0), \quad \text { in } \Omega
$$

then with $v_{0}=\gamma\left(u_{0}\right)$ we have

$$
u^{0} \in C\left([0, T), H^{1}(\Omega)\right), \quad u^{0}(0)=u_{0} .
$$


REMARK 2.6. Note that in (2.13) the time derivative is taken in distributional sense. In particular, for any $T>0$ and any smooth test function $\varphi(t, x)$ in $[0, T] \times \bar{\Omega}$ such that $\varphi(T, \cdot)=0$ we have that [2.13) is satisfied in the sense that

$$
\begin{aligned}
& -\int_{\Gamma} v_{0} \varphi(0, \cdot)-\int_{0}^{T} \int_{\Gamma} u^{0} \varphi_{t}+\int_{0}^{T}\left[\int_{\Omega} \nabla u^{0} \nabla \varphi+\lambda \int_{\Omega} u^{0} \varphi\right] \\
& +\int_{0}^{T} \int_{\Gamma} V_{0} u^{0} \varphi=\int_{0}^{T} \int_{\Omega} f \varphi+\int_{0}^{T} \int_{\Gamma} g \varphi .
\end{aligned}
$$

REMARK 2.7. Note that given for $f \in L^{2}\left((0, T), L^{2}(\Omega)\right)$ and $g \in L^{2}((0, T) \times$ $\Gamma)$, for 2.13) uniquenes of solutions holds in the class $L^{2}\left((0, T), H^{1}(\Omega)\right)$. Indeed for such a solution satisfying $u^{0}(0)=0, f=0=g$, we have $\gamma\left(u^{0}\right) \in$ $L^{2}\left((0, T), H^{1 / 2}(\Gamma)\right)$ while (2.13) gives $\gamma\left(u^{0}\right)_{t} \in L^{2}\left((0, T), H^{-1 / 2}(\Gamma)\right)$. Hence $\gamma\left(u^{0}\right) \in$ $C\left([0, T], L^{2}(\Gamma)\right)$, the function $t \rightarrow\left\|u^{0}(t)\right\|_{L^{2}(\Gamma)}^{2}$ is absolutely continuous and its time derivative is $2 \int_{\Gamma} u^{0}(t) u_{t}^{0}(t)$. Hence, taking $u^{0}(t)$ as a test function in (2.13) and using Gronwall's inequality we get $u^{0}=0$ in $[0, T]$.

\section{Concentrating integrals}

In this section we show several results that describe how different concentrated integrals converge to surface integrals. Hereafter we denote by $C>0$ any positive constant such that $C$ is independent of $\varepsilon$ and $t$. This constant may change from line to line.

The following lemma a particular case of a result proved in 2] and basically states that concentrated functions behave as traces.

Lemma 3.1. A) Assume that $v \in H^{s}(\Omega)$ with $s>\frac{1}{2}$ and such that $H^{s}(\Omega) \subset$ $L^{q}(\Gamma)$, i.e. $s-\frac{N}{2} \geq-\frac{(N-1)}{q}$. Then for sufficiently small $\varepsilon_{0}$, we have, for some positive constant $C$ independent of $\varepsilon$,

$$
\frac{1}{\varepsilon} \int_{\omega_{\varepsilon}}|v|^{q} \leq C\|v\|_{H^{s}(\Omega)}^{q}
$$

and

$$
\lim _{\varepsilon \rightarrow 0} \frac{1}{\varepsilon} \int_{\omega_{\varepsilon}}|v|^{q}=\int_{\Gamma}|v|^{q} .
$$

B) Consider a family $f_{\varepsilon}$ defined on $\omega_{\varepsilon}$, such that for some $1 \leq r<\infty$ and a positive constant $C$ independent of $\varepsilon$,

$$
\frac{1}{\varepsilon} \int_{\omega_{\varepsilon}}\left|f_{\varepsilon}\right|^{r} \leq C
$$

Then, for every sequence converging to zero (that we still denote $\varepsilon \rightarrow 0$ ) there exists a subsequence (that we still denote the same) and a function $f_{0} \in L^{r}(\Gamma)$ (or a bounded Radon measure on $\Gamma, f_{0} \in \mathcal{M}(\Gamma)$ if $r=1$ ) such that, for every $s>\frac{1}{2}$ and

$$
s-\frac{N}{2}>-\frac{N-1}{r^{\prime}}
$$

we have that

$$
\frac{1}{\varepsilon} \mathcal{X}_{\omega_{\varepsilon}} f_{\varepsilon} \rightarrow f_{0} \quad \text { in } H^{-s}(\Omega) \text { as } \varepsilon \rightarrow 0
$$


where $\mathcal{X}_{\omega_{\varepsilon}}$ is the characteristic function of the set $\omega_{\varepsilon}$. In particular, for any smooth function $\varphi$, defined in $\bar{\Omega}$, we have

$$
\lim _{\varepsilon \rightarrow 0} \frac{1}{\varepsilon} \int_{\omega_{\varepsilon}} f_{\varepsilon} \varphi=\int_{\Gamma} f_{0} \varphi .
$$

Moreover, if $u^{\varepsilon} \rightarrow u^{0}$ weakly in $H^{s}(\Omega)$ or strongly in case of equal sign in (3.3), then

$$
\lim _{\varepsilon \rightarrow 0} \frac{1}{\varepsilon} \int_{\omega_{\varepsilon}} f_{\varepsilon} u^{\varepsilon}=\int_{\Gamma} f_{0} u^{0} .
$$

In particular, assume $\varphi \in H^{\sigma}(\Omega)$ with $\sigma>\frac{1}{2}$, and denote $\varphi_{0}$ the trace of $\varphi$ on Г. Then

$$
\frac{1}{\varepsilon} \mathcal{X}_{\omega_{\varepsilon}} \varphi \rightarrow \varphi_{0} \quad \text { in } H^{-s}(\Omega) \text { as } \varepsilon \rightarrow 0
$$

for any $s$ such that $s>\frac{1}{2}$ and

$$
\left(s-\frac{N}{2}\right)_{-}+\left(\sigma-\frac{N}{2}\right)_{-}>-N+1,
$$

where $x_{-}$denotes the negative part of $x$. Finally if $\varphi \in C(\bar{\Omega})$, 3.4 holds for any $s>\frac{1}{2}$.

Also the following consequence will be used further below.

Corollary 3.2. Assume

$$
\left\|u_{0}^{\varepsilon}\right\|_{H^{1}(\Omega)}^{2} \leq C .
$$

Then, by taking subsequences if necessary, there exists $u_{0} \in H^{1}(\Omega)$ such that, as $\varepsilon \rightarrow 0$,

$$
u_{0}^{\varepsilon} \rightarrow u_{0} \quad \text { weakly in } H^{1}(\Omega), \quad \frac{1}{\varepsilon} \mathcal{X}_{\omega_{\varepsilon}} u_{0}^{\varepsilon} \rightarrow u_{0 \mid \Gamma} \quad \text { weakly in } H^{-1}(\Omega)
$$

and

$$
\lim _{\varepsilon \rightarrow 0} \frac{1}{\varepsilon} \int_{\omega_{\varepsilon}}\left|u_{0}^{\varepsilon}\right|^{2}=\int_{\Gamma}\left|u_{0}\right|^{2} .
$$

Proof From part A) in Lemma 3.1 with $q=2$, we have

$$
\frac{1}{\varepsilon} \int_{\omega_{\varepsilon}}\left|u_{0}^{\varepsilon}\right|^{2} \leq C\left\|u_{0}^{\varepsilon}\right\|_{H^{1}(\Omega)}^{2} \leq C .
$$

Hence there exists $u_{0} \in H^{1}(\Omega)$ such that, as $\varepsilon \rightarrow 0, u_{0}^{\varepsilon} \rightarrow u_{0}$ weakly in $H^{1}(\Omega)$ and by part B) in Lemma 3.1 with $r=2$, there exists $v_{0} \in L^{2}(\Gamma)$ such that $\frac{1}{\varepsilon} \mathcal{X}_{\omega_{\varepsilon}} u_{0}^{\varepsilon} \rightarrow v_{0}$ in $H^{-1}(\Omega)$.

Since (3.3) is satisfied with $s=1, r=2$, again part B) in Lemma 3.1 implies that

$$
\lim _{\varepsilon \rightarrow 0} \frac{1}{\varepsilon} \int_{\omega_{\varepsilon}}\left|u_{0}^{\varepsilon}\right|^{2}=\int_{\Gamma} u_{0} v_{0} .
$$

Therefore it remains to prove that $v_{0}=u_{0 \mid \Gamma}$. For this note that if $\varphi \in H^{1}(\Omega)$ we have, by (3.4), (3.5) with $s=1=\sigma$,

$$
\varphi_{\varepsilon}=\frac{1}{\varepsilon} \mathcal{X}_{\omega_{\varepsilon}} \varphi \rightarrow \varphi_{\mid \Gamma} \quad \text { in } H^{-1}(\Omega)
$$


Then $\left\langle u_{0}^{\varepsilon}, \varphi_{\varepsilon}\right\rangle=\frac{1}{\varepsilon} \int_{\omega_{\varepsilon}} u_{0}^{\varepsilon} \varphi$ and the left hand side converges to $\left\langle u_{0}, \varphi_{0}\right\rangle=\int_{\Gamma} u_{0} \varphi$ while the right hand side converges to $\left\langle v_{0}, \varphi\right\rangle=\int_{\Gamma} v_{0} \varphi$. Hence, $v_{0}=u_{0 \mid \Gamma}$ as claimed.r

Lemma 3.1 can now be extended to handle concentrating integrals including a time dependence.

Lemma 3.3. A) Consider $v \in L^{r}\left((0, T), H^{s}(\Omega)\right)$ with $1 \leq r<\infty, s>\frac{1}{2}$ and $H^{s}(\Omega) \subset L^{q}(\Gamma)$, that is, $s-\frac{N}{2} \geq-\frac{(N-1)}{q}$. Then,

$$
\int_{0}^{T}\left(\frac{1}{\varepsilon} \int_{\omega_{\varepsilon}}|v|^{q}\right)^{r / q} \leq C \int_{0}^{T}\|v(t, \cdot)\|_{H^{s}(\Omega)}^{r} d t=\|v\|_{L^{r}\left((0, T), H^{s}(\Omega)\right)}^{r}
$$

and

$$
\lim _{\varepsilon \rightarrow 0} \int_{0}^{T}\left(\frac{1}{\varepsilon} \int_{\omega_{\varepsilon}}|v|^{q}\right)^{r / q}=\int_{0}^{T}\left(\int_{\Gamma}|v|^{q}\right)^{r / q}=\|v\|_{L^{r}\left((0, T), L^{q}(\Gamma)\right)}^{r} .
$$

B) Consider a family $g_{\varepsilon}$ defined on $(0, T) \times \omega_{\varepsilon}$, such that for some $1<q<\infty$, $1 \leq r<\infty$ and a positive constant $C$ independent of $\varepsilon$,

$$
\int_{0}^{T}\left(\frac{1}{\varepsilon} \int_{\omega_{\varepsilon}}\left|g_{\varepsilon}(t, x)\right|^{r} d x\right)^{\frac{q}{r}} d t \leq C
$$

or $\int_{0}^{T} \sup _{x \in \omega_{\varepsilon}}\left|g_{\varepsilon}(t, x)\right|^{q} d t \leq C$ for the case $r=\infty$.

Then, for every s satisfying (3.3), and for every sequence converging to zero (that we still denote $\varepsilon \rightarrow 0$ ) there exists a subsequence (that we still denote the same) and a function $g \in L^{q}\left((0, T), L^{r}(\Gamma)\right)$ (or a bounded Radon measure on $\Gamma, g \in$ $L^{q}((0, T), \mathcal{M}(\Gamma))$ if $\left.r=1\right)$ such that

$$
\frac{1}{\varepsilon} \mathcal{X}_{\omega_{\varepsilon}} g_{\varepsilon} \rightarrow g \quad \text { in } L^{q}\left((0, T), H^{-s}(\Omega)\right), \text { weakly as } \varepsilon \rightarrow 0,
$$

where $\mathcal{X}_{\omega_{\varepsilon}}$ is the characteristic function of the set $\omega_{\varepsilon}$. In particular, for any smooth function $\varphi$, defined in $[0, T] \times \bar{\Omega}$, we have

$$
\lim _{\varepsilon \rightarrow 0} \frac{1}{\varepsilon} \int_{0}^{T} \int_{\omega_{\varepsilon}} g_{\varepsilon} \varphi=\int_{0}^{T} \int_{\Gamma} g \varphi .
$$

Also, if $u^{\varepsilon} \rightarrow u^{0}$ strongly in $L^{q^{\prime}}\left((0, T), H^{s}(\Omega)\right)$ then

$$
\lim _{\varepsilon \rightarrow 0} \frac{1}{\varepsilon} \int_{0}^{T} \int_{\omega_{\varepsilon}} g_{\varepsilon} u^{\varepsilon}=\int_{0}^{T} \int_{\Gamma} g u^{0} .
$$

C) Consider a family $g_{\varepsilon}$ defined on $(0, T) \times \omega_{\varepsilon}$, and assume that for some $1<r, q<\infty$, there exist $h \in L^{q}(0, T)$, and $g \in L^{q}\left((0, T), L^{r}(\Gamma)\right)$ such that

$$
\begin{gathered}
\left(\frac{1}{\varepsilon} \int_{\omega_{\varepsilon}}\left|g_{\varepsilon}(t, \cdot)\right|^{r}\right)^{\frac{1}{r}} \leq h(t), \quad \text { a.e. } \quad t \in[0, T] \\
\frac{1}{\varepsilon} \mathcal{X}_{\omega_{\varepsilon}} g_{\varepsilon}(t, \cdot) \rightarrow g(t, \cdot) \text { in } H^{-s}(\Omega) \quad \text { a.e. } \quad t \in(0, T)
\end{gathered}
$$

with s satisfying (3.3). Then

$$
\frac{1}{\varepsilon} \mathcal{X}_{\omega_{\varepsilon}} g_{\varepsilon} \rightarrow g \quad \text { in } L^{q}\left((0, T), H^{-s}(\Omega)\right) .
$$


In particular, if $\varphi \in L^{q}\left((0, T), H^{\sigma}(\Omega)\right)$, with $\sigma>\frac{1}{2}$, we consider $\varphi_{\varepsilon}(t)=$ $\frac{1}{\varepsilon} \mathcal{X}_{\omega_{\varepsilon}} \varphi(t)$ and $\varphi_{0}(t)=\left.\varphi\right|_{\Gamma}(t)$. Then

$$
\frac{1}{\varepsilon} \mathcal{X}_{\omega_{\varepsilon}} \varphi \rightarrow \varphi_{0} \quad \text { in } L^{q}\left((0, T), H^{-s}(\Omega)\right) \text { as } \varepsilon \rightarrow 0
$$

for $\sigma, s$ as in (3.5). If $\varphi \in C([0, T] \times \bar{\Omega})$, (3.15) holds for any $q>1$ and $s>\frac{1}{2}$.

Proof A) Observe that (3.1) gives (3.6) right away. Now, we note that for fixed $t \in[0, T]$, from (3.2) we get

$$
\left(\frac{1}{\varepsilon} \int_{\omega_{\varepsilon}}|v(t, \cdot)|^{q}\right)^{r / q} \leq C\|v(t, \cdot)\|_{H^{s}(\Omega)}^{r} \text { and } \lim _{\varepsilon \rightarrow 0} \frac{1}{\varepsilon} \int_{\omega_{\varepsilon}}|v(t, \cdot)|^{q}=\int_{\Gamma}|v(t, \cdot)|^{q} .
$$

Then, applying Lebesgue's dominated convergence theorem, we obtain (3.7).

B) Define, for $s$ satisfying (3.3), the linear forms

$$
L_{\varepsilon}(\varphi)=\frac{1}{\varepsilon} \int_{0}^{T} \int_{\omega_{\varepsilon}} g_{\varepsilon} \varphi
$$

on $L^{q^{\prime}}\left((0, T), H^{s}(\Omega)\right)$. By Hölder's inequality we get

$$
\begin{aligned}
& \left|L_{\varepsilon}(\varphi)\right| \leq \int_{0}^{T}\left(\frac{1}{\varepsilon} \int_{\omega_{\varepsilon}}\left|g_{\varepsilon}\right|^{r}\right)^{\frac{1}{r}}\left(\frac{1}{\varepsilon} \int_{\omega_{\varepsilon}}|\varphi|^{r^{\prime}}\right)^{\frac{1}{r^{\prime}}} \\
& \leq\left[\int_{0}^{T}\left(\frac{1}{\varepsilon} \int_{\omega_{\varepsilon}}\left|g_{\varepsilon}\right|^{r}\right)^{\frac{q}{r}}\right]^{\frac{1}{q}}\left[\int_{0}^{T}\left(\frac{1}{\varepsilon} \int_{\omega_{\varepsilon}}|\varphi|^{r^{\prime}}\right)^{\frac{q^{\prime}}{r^{\prime}}}\right]^{\frac{1}{q^{\prime}}} .
\end{aligned}
$$

Hence using (3.8) and (3.6), we get

$$
\left|L_{\varepsilon}(\varphi)\right| \leq C\left[\int_{0}^{T}\left(\frac{1}{\varepsilon} \int_{\omega_{\varepsilon}}|\varphi|^{r^{\prime}}\right)^{\frac{q^{\prime}}{r^{\prime}}}\right]^{\frac{1}{q^{\prime}}} \leq C\|\varphi\|_{L^{q^{\prime}}\left((0, T), H^{s}(\Omega)\right)} .
$$

Hence $L_{\varepsilon}$ is a bounded family in the dual space of $L^{q^{\prime}}\left((0, T), H^{s}(\Omega)\right)$. Therefore, by the Banach-Alaouglu-Bourbaki theorem, and taking subsequences if necessary, we have that there exists $L_{0} \in\left[L^{q^{\prime}}\left((0, T), H^{s}(\Omega)\right)\right]^{\prime}:=L^{q}\left((0, T), H^{-s}(\Omega)\right)$ such that

$$
L_{\varepsilon}(\varphi) \rightarrow L_{0}(\varphi), \quad \text { for all } \varphi \in L^{q^{\prime}}\left((0, T), H^{s}(\Omega)\right)
$$

as $\varepsilon \rightarrow 0$ and the limit is uniform for $\varphi$ in compact sets of $L^{q^{\prime}}\left((0, T), H^{s}(\Omega)\right)$.

In particular, from the first inequality in (3.16) and (3.7), we get

$$
\left|L_{0}(\varphi)\right| \leq C\|\varphi\|_{\left.L^{q^{\prime}}\left((0, T), L^{r^{\prime}}(\Gamma)\right)\right)} \quad \text { for every } \varphi \in L^{q^{\prime}}\left((0, T), H^{s}(\Omega)\right) .
$$

Now taking into account that if $X \subset Y$ is dense, then $L^{q^{\prime}}((0, T), X)$ is dense in $L^{q^{\prime}}((0, T), Y)$ and since traces of $H^{s}(\Omega)$ are dense in $L^{r^{\prime}}(\Gamma)$, we get

$$
L^{q^{\prime}}\left((0, T), H^{s}(\Omega)\right) \text { is dense in } L^{q^{\prime}}\left((0, T), L^{r^{\prime}}(\Gamma)\right) .
$$

Thus, $L_{0} \in\left(L^{q^{\prime}}\left((0, T), L^{r^{\prime}}(\Gamma)\right)\right)^{\prime}$ and then there exists $g \in L^{q}\left((0, T), L^{r}(\Gamma)\right)$ such that $L_{0}=g$, i.e.

$$
L_{0}(\varphi)=\int_{0}^{T} \int_{\Gamma} g \varphi
$$

which proves (3.9), (3.10) and (3.11). 
C) First, we note that from (3.12) together with (3.1) we have that for $\varphi \in$ $H^{s}(\Omega)$

$$
\left|\frac{1}{\varepsilon} \int_{\omega_{\varepsilon}} g_{\varepsilon}(t, \cdot) \varphi\right| \leq\left[\frac{1}{\varepsilon} \int_{\omega_{\varepsilon}}\left|g_{\varepsilon}(t, \cdot)\right|^{r}\right]^{\frac{1}{r}}\left[\frac{1}{\varepsilon} \int_{\omega_{\varepsilon}}|\varphi|^{r^{\prime}}\right]^{\frac{1}{r^{\prime}}} \leq C h(t)\|\varphi\|_{H^{s}(\Omega)},
$$

that is

$$
\left\|\frac{1}{\varepsilon} \mathcal{X}_{\omega_{\varepsilon}} g_{\varepsilon}(t, \cdot)\right\|_{H^{-s}(\Omega)} \leq C h(t)
$$

Next, taking into account (3.13) we can apply Lebesgue's dominated convergence theorem to get (3.14).

In particular, if $\varphi \in L^{q}\left((0, T), H^{\sigma}(\Omega)\right)$, with $\sigma>\frac{1}{2}$, we consider $g_{\varepsilon}(t)=$ $\frac{1}{\varepsilon} \mathcal{X}_{\omega_{\varepsilon}} \varphi(t)$ and $\varphi_{0}(t)=\left.\varphi\right|_{\Gamma}(t)$. Then, by [3.1), we have for a.e. $t \in(0, T)$

$$
\left(\frac{1}{\varepsilon} \int_{\omega_{\varepsilon}}|\varphi(t, \cdot)|^{r}\right)^{1 / r} \leq C\|\varphi(t, \cdot)\|_{H^{\sigma}(\Omega)}=h(t) \in L^{q}(0, T)
$$

and by (3.4),

$$
\lim _{\varepsilon \rightarrow 0} \frac{1}{\varepsilon} \mathcal{X}_{\omega_{\varepsilon}} \varphi(t) \rightarrow \varphi_{0}(t) \quad \text { in } H^{-s}(\Omega) \text { as } \varepsilon \rightarrow 0
$$

for $\sigma, s$ as in (3.5). Then (3.12) and (3.13) are satisfied.

If $\varphi \in C([0, T] \times \bar{\Omega})$, denote $h(t)=\sup _{x \in \bar{\Omega}}|\varphi(t, x)|$. Then for any $1 \leq r, q<\infty$, taking into account that $\left|\omega_{\varepsilon}\right| \leq C \varepsilon$ for some $C>0$, we obtain

$$
\left(\frac{1}{\varepsilon} \int_{\omega_{\varepsilon}}|\varphi(t, x)|^{r} d x\right)^{\frac{1}{r}} \leq C h(t) \in L^{q}(0, T) .
$$

Also, for fixed $t \in[0, T]$, by (3.4) we have

$$
\frac{1}{\varepsilon} \mathcal{X}_{\omega_{\varepsilon}} \varphi(t, \cdot) \rightarrow \varphi_{0}(t, \cdot), \quad \text { as } \varepsilon \rightarrow 0, \text { in } H^{-s}(\Omega) .
$$

for any $s>\frac{1}{2}$. Then, we can choose $r>1$ such that $s-\frac{N}{2}>-\frac{N-1}{r^{\prime}}$ and then (3.12) and (3.13) are satisfied again.

REMARK 3.4. The results in parts, B) and C) of Lemma 3.3 also hold with minor changes when either $r=1$ or $q=1$. Since in the proof above $L^{q^{\prime}}$ and $L^{r^{\prime}}$ appear, in such a case some spaces of measures enter in the result. Also, when, $\varphi \in C([0, T] \times \bar{\Omega})$ it can be actually shown that [3.15) holds for $r=\infty$.

For the sake of simplicity in the exposition we have not included these cases.

Now we prove the following result that will be used below in the analysis of (1.1) and (1.2). Note that the assumption on the potentials below is, not only uniform in $\varepsilon$, but more restrictive in $\rho$ than the one needed for fixed $\varepsilon$, as in (2.2), i.e. $\rho>N / 2$.

Lemma 3.5. Assume that the potentials $V_{\varepsilon}$ satisfy

$$
\frac{1}{\varepsilon} \int_{\omega_{\varepsilon}}\left|V_{\varepsilon}\right|^{\rho} \leq C, \text { with } \rho>N-1
$$

and assume, that after taking some subsequence, if necessary, we have

$$
\lim _{\varepsilon \rightarrow 0} \frac{1}{\varepsilon} \int_{\omega_{\varepsilon}} V_{\varepsilon} \varphi=\int_{\Gamma} V \varphi
$$

for any smooth function $\varphi$ defined in $\bar{\Omega}$ and for some function $V \in L^{\rho}(\Gamma)$; see Lemma 3.1, part B). Then 
i) If we denote by $\left(V_{\varepsilon}\right)_{-}$the negative part of the potential, then we have that for any $\delta>0$ there exists $C_{\delta}>0$, independent of $\varepsilon>0$ such that

$$
\frac{1}{\varepsilon} \int_{\omega_{\varepsilon}}\left(V_{\varepsilon}\right)_{-}|\phi|^{2} \leq \delta\|\phi\|_{H^{1}(\Omega)}^{2}+C_{\delta}\|\phi\|_{L^{2}(\Omega)}^{2}
$$

and

$$
\frac{1}{\varepsilon} \int_{\omega_{\varepsilon}}\left(V_{\varepsilon}\right)_{-}|\phi|^{2} \leq \delta\|\phi\|_{H^{1}(\Omega)}^{2}+\frac{C_{\delta}}{\varepsilon} \int_{\omega_{\varepsilon}}|\phi|^{2} .
$$

ii) Analogously, for any $\delta>0$ there exists $C_{\delta}>0$, such that

$$
\int_{\Gamma}(V)_{-}|\phi|^{2} \leq \delta\|\phi\|_{H^{1}(\Omega)}^{2}+C_{\delta}\|\phi\|_{L^{2}(\Omega)}^{2}
$$

and

$$
\int_{\Gamma}(V)_{-}|\phi|^{2} \leq \delta\|\phi\|_{H^{1}(\Omega)}^{2}+C_{\delta} \int_{\Gamma}|\phi|^{2} .
$$

iii) There exists some $\lambda_{0} \in \mathbb{R}$, independent of $\varepsilon>0$, such that for $\lambda>\lambda_{0}$ the elliptic operator, associated to the parabolic problems (1.1) and (1.2), are positive.

iv) If $s$ is such that $\frac{1}{2}+\frac{N-1}{2 \rho}<s \leq 1$ and

$$
u^{\varepsilon} \rightarrow u^{0} \text { weakly in } L^{2}\left((0, T), H^{s}(\Omega)\right),
$$

then for any function $\varphi \in L^{2}\left((0, T), H^{s}(\Omega)\right)$ we have

$$
\frac{1}{\varepsilon} \int_{0}^{T} \int_{\omega_{\varepsilon}} V_{\varepsilon} u^{\varepsilon} \varphi \rightarrow \int_{0}^{T} \int_{\Gamma} V u^{0} \varphi
$$

Proof:

i) Note that for every $\phi \in H^{1}(\Omega)$ we have the bound

$$
\frac{1}{\varepsilon} \int_{\omega_{\varepsilon}}\left(V_{\varepsilon}\right)_{-}|\phi|^{2} \leq\left(\frac{1}{\varepsilon} \int_{\omega_{\varepsilon}}\left|\left(V_{\varepsilon}\right)_{-}\right|^{\rho}\right)^{\frac{1}{\rho}}\left[\frac{1}{\varepsilon} \int_{\omega_{\varepsilon}}|\phi|^{2 \rho^{\prime}}\right]^{\frac{1}{\rho}} \leq C\left[\frac{1}{\varepsilon} \int_{\omega_{\varepsilon}}|\phi|^{2 \rho^{\prime}}\right]^{\frac{1}{\rho^{\prime}}} .
$$

Now, since $\rho>N-1$, there exists $\frac{N-1}{2 \rho}+\frac{1}{2} \leq s<1$ such that $H^{s}(\Omega) \subset L^{2 \rho^{\prime}}(\Gamma)$ and from part A) in Lemma 3.1 and interpolation, we have that

$$
\frac{1}{\varepsilon} \int_{\omega_{\varepsilon}}\left(V_{\varepsilon}\right)_{-}|\phi|^{2} \leq C\|\phi\|_{H^{s}(\Omega)}^{2} \leq C\|\phi\|_{H^{1}(\Omega)}^{2 s}\|\phi\|_{L^{2}(\Omega)}^{2(1-s)} .
$$

Finally using Young's inequality, we get for any $\delta>0$

$$
\frac{1}{\varepsilon} \int_{\omega_{\varepsilon}}\left(V_{\varepsilon}\right)_{-}|\phi|^{2} \leq \delta\|\phi\|_{H^{1}(\Omega)}^{2}+C_{\delta}\|\phi\|_{L^{2}(\Omega)}^{2}
$$

and we get the first inequality.

For the second one, observe that starting from (3.17), using $\rho>N-1$ and interpolating the Lebesgue norms in $\omega_{\varepsilon}$ we get

$$
\frac{1}{\varepsilon} \int_{\omega_{\varepsilon}}\left(V_{\varepsilon}\right)_{-}|\phi|^{2} \leq \frac{1}{\varepsilon^{1 / \rho^{\prime}}}\|\phi\|_{L^{2^{* *}\left(\omega_{\varepsilon}\right)}}^{2 \theta}\|\phi\|_{L^{2}\left(\omega_{\varepsilon}\right)}^{2(1-\theta)}
$$

where $2^{* *}=\frac{2(N-1)}{N-2}$ and $\theta \in(0,1)$ such that $\frac{1}{2 \rho^{\prime}}=\frac{\theta}{2^{* *}}+\frac{1-\theta}{2}$. Using this last condition and splitting the term with $\varepsilon$ between the two integrals above, Young's inequality leads, for any $\delta>0$ and $C_{\delta}$ independent of $\varepsilon$, to

$$
\frac{1}{\varepsilon} \int_{\omega_{\varepsilon}}\left(V_{\varepsilon}\right)_{-}|\phi|^{2} \leq \frac{\delta}{\varepsilon^{2 / 2^{* *}}}\|\phi\|_{L^{2^{* *}}\left(\omega_{\varepsilon}\right)}^{2}+\frac{C_{\delta}}{\varepsilon}\|\phi\|_{L^{2}\left(\omega_{\varepsilon}\right)}^{2} .
$$


The right hand side above is

$$
\delta\left(\frac{1}{\varepsilon} \int_{\omega_{\varepsilon}}|\phi|^{2^{* *}}\right)^{2 / 2^{* *}}+C_{\delta} \frac{1}{\varepsilon} \int_{\omega_{\varepsilon}}|\phi|^{2}
$$

which, by (3.1) can be bounded by

$$
\delta\|\phi\|_{H^{1}(\Omega)}^{2}+\frac{C_{\delta}}{\varepsilon} \int_{\omega_{\varepsilon}}|\phi|^{2}
$$

which proves the claim.

ii) A similar argument using that $V \in L^{\rho}(\Gamma)$ and $\rho>N-1$ gives an estimate completely similar to (3.17), now with boundary integrals. The rest also follows as above but using boundary integrals instead of concentrated integrals.

iii) Using parts i) and ii) it is clear that there exists $\lambda_{0}$ such that the bilinear forms in $H^{1}(\Omega)$

$$
a_{\varepsilon}(\phi, \xi)=\int_{\Omega} \nabla \phi \nabla \xi+\lambda \int_{\Omega} \phi \xi+\frac{1}{\varepsilon} \int_{\omega_{\varepsilon}} V_{\varepsilon} \phi \xi
$$

and

$$
a_{0}(\phi, \xi)=\int_{\Omega} \nabla \phi \nabla \xi+\lambda \int_{\Omega} \phi \xi+\int_{\Gamma} V \phi \xi
$$

are uniformly coercive for $\lambda>\lambda_{0}$. In fact, $a_{\varepsilon}(\phi, \phi), a_{0}(\phi, \phi)$ can be bounded below by

$$
(1-\delta) \int_{\Omega}|\nabla \phi|^{2}+\left(\lambda-\delta-C_{\delta}\right) \int_{\Omega}|\phi|^{2} .
$$

iii) First, for $s, \sigma>\frac{1}{2}$ and $\left(s-\frac{N}{2}\right)_{-}+\left(\sigma-\frac{N}{2}\right)_{-}>-\frac{N-1}{\rho^{\prime}}$, we define the operators, $P_{\varepsilon}: H^{s}(\Omega) \rightarrow H^{-\sigma}(\Omega)$ for $0 \leq \varepsilon \leq \varepsilon_{0}$ by

$$
<P_{\varepsilon}(u), \varphi>=\frac{1}{\varepsilon} \int_{\omega_{\varepsilon}} V_{\varepsilon} u \varphi, \quad<P_{0}(u), \varphi>=\int_{\Gamma} V u \varphi .
$$

Then from Lemma 2.5 in 2 we get $P_{\varepsilon} \rightarrow P_{0}$ in $\mathcal{L}(X, Y)$ with $X=H^{s}(\Omega)$ and $Y=H^{-\sigma}(\Omega)$.

Now we consider $\sigma=s$. This choice is possible provided $2\left(s-\frac{N}{2}\right)_{-}>-\frac{N-1}{\rho^{\prime}}$, which leads to the lower bound on $s$ in the statement. Note that this lower bound is compatible with $s \leq 1$ because $\rho>N-1$.

Then, by Lemma 3.6] below, we have that $P_{\varepsilon} u^{\varepsilon} \rightarrow P_{0} u^{0}$ weakly in $L^{2}((0, T), Y)$. In particular for any function $\varphi \in L^{2}\left((0, T), Y^{\prime}\right)=L^{2}\left((0, T), H^{s}(\Omega)\right)$ we have

$$
\frac{1}{\varepsilon} \int_{0}^{T} \int_{\omega_{\varepsilon}} V_{\varepsilon} u^{\varepsilon} \varphi \rightarrow \int_{0}^{T} \int_{\Gamma} V u^{0} \varphi
$$

and we conclude

Now we prove the result used above.

Lemma 3.6. Assume $X$ and $Y$ are reflexive Banach spaces and $P_{\varepsilon} \rightarrow P_{0}$ in $\mathcal{L}(X, Y)$.

If $u^{\varepsilon} \rightarrow u^{0}$ weakly in $L^{2}((0, T), X)$, then

$$
P_{\varepsilon} u^{\varepsilon} \rightarrow P_{0} u^{0} \text { weakly in } L^{2}((0, T), Y) \text {. }
$$


Proof First note that if $\int_{0}^{T}\left\|u^{\varepsilon}(t)\right\|_{X}^{2}=\left\|u^{\varepsilon}\right\|_{L^{2}((0, T), X)}^{2} \leq C$ then

$$
\begin{aligned}
& \left\|P_{\varepsilon} u^{\varepsilon}-P_{0} u^{\varepsilon}\right\|_{L^{2}((0, T), Y)}^{2} \leq \int_{0}^{T}\left\|P_{\varepsilon}-P_{0}\right\|_{\mathcal{L}(X, Y)}^{2}\left\|u^{\varepsilon}(t)\right\|_{X}^{2} d t \\
& \leq C\left\|P_{\varepsilon}-P_{0}\right\|_{\mathcal{L}(X, Y)}^{2} \rightarrow 0 \text { as } \varepsilon \rightarrow 0 .
\end{aligned}
$$

Now assume $u^{\varepsilon} \rightarrow u^{0}$ weakly in $L^{2}((0, T), X)$, and take $\phi \in L^{2}\left((0, T), Y^{\prime}\right)$, then

$$
\begin{gathered}
\left|\int_{0}^{T}<P_{\varepsilon} u^{\varepsilon}, \phi>_{Y, Y^{\prime}}-<P_{0} u^{0}, \phi>_{Y, Y^{\prime}}\right| \leq \\
\leq\left|\int_{0}^{T}<P_{\varepsilon} u^{\varepsilon}, \phi>_{Y, Y^{\prime}} \pm<P_{0} u^{\varepsilon}, \phi>_{Y, Y^{\prime}}-<P_{0} u^{0}, \phi>_{Y, Y^{\prime}}\right| \leq(1)+(2)
\end{gathered}
$$

where

and

$$
(1) \equiv\left|\int_{0}^{T}<P_{\varepsilon} u^{\varepsilon}, \phi>_{Y, Y^{\prime}}-P_{0} u^{\varepsilon}, \phi>_{Y, Y^{\prime}}\right|
$$

$$
(2) \equiv\left|\int_{0}^{T}<P_{0} u^{\varepsilon}, \phi>_{Y, Y^{\prime}}-<P_{0} u^{0}, \phi>_{Y, Y^{\prime}}\right| .
$$

Thus, we obtain

$$
(1) \leq\left|\int_{0}^{T}<P_{\varepsilon} u^{\varepsilon}-P_{0} u^{\varepsilon}, \phi>_{Y, Y^{\prime}}\right| \leq \int_{0}^{T}\left\|P_{\varepsilon} u^{\varepsilon}-P_{0} u^{\varepsilon}\right\|_{Y}\|\phi\|_{Y^{\prime}} d t
$$

and we get $(1) \rightarrow 0$ as $\varepsilon \rightarrow 0$. Moreover, we have that

$$
(2) \leq\left|\int_{0}^{T}<P_{0}\left(u^{\varepsilon}-u^{0}\right), \phi>_{Y, Y^{\prime}}\right|=\left|\int_{0}^{T}<u^{\varepsilon}-u^{0}, P_{0}^{*} \phi>_{X, X^{\prime}}\right|
$$

with $P_{0}^{*} \phi \in L^{2}\left((0, T), X^{\prime}\right)$. Then using $u^{\varepsilon} \rightarrow u^{0}$ weakly in $L^{2}((0, T), X)$ we get also $(2) \rightarrow 0$ as $\varepsilon \rightarrow 0$

We also have the following result.

Lemma 3.7. We consider a family of functions $u^{\varepsilon}:[0, T] \rightarrow H^{1}(\Omega)$ such that for some positive constant $C$ independent of $\varepsilon$ and $t$, we have

$$
\left\|u^{\varepsilon}(t, \cdot)\right\|_{H^{1}(\Omega)} \leq C, \quad t \in[0, T]
$$

and $u_{t}^{\varepsilon} \in L^{2}\left((0, T) \times \omega_{\varepsilon}\right)$ with

$$
\frac{1}{\varepsilon} \int_{0}^{T} \int_{\omega_{\varepsilon}}\left|u_{t}^{\varepsilon}\right|^{2} \leq C
$$

Then, there exists a subsequence (that we still denote the same) and a function $u^{0} \in L^{\infty}\left((0, T), H^{1}(\Omega)\right)$ with $u_{\mid \Gamma}^{0} \in H^{1}\left((0, T), L^{2}(\Gamma)\right)$ such that as $\varepsilon \rightarrow 0$,

$$
u^{\varepsilon} \rightarrow u^{0} \quad w-* \quad \text { in } L^{\infty}\left((0, T), H^{1}(\Omega)\right)
$$

and

$$
\frac{1}{\varepsilon} \mathcal{X}_{\omega_{\varepsilon}} u^{\varepsilon} \rightarrow u_{\mid \Gamma}^{0} \quad \text { in } H^{1}\left((0, T), H^{-1}(\Omega)\right) .
$$

In particular, for every $\varphi \in L^{2}\left((0, T), H^{1}(\Omega)\right)$ we have

$$
\lim _{\varepsilon \rightarrow 0} \frac{1}{\varepsilon} \int_{0}^{T} \int_{\omega_{\varepsilon}} u^{\varepsilon} \varphi=\int_{0}^{T} \int_{\Gamma} u^{0} \varphi
$$




$$
\lim _{\varepsilon \rightarrow 0} \frac{1}{\varepsilon} \int_{0}^{T} \int_{\omega_{\varepsilon}} u_{t}^{\varepsilon} \varphi=\int_{0}^{T} \int_{\Gamma} u_{t}^{0} \varphi
$$

Finally

$$
\frac{1}{\varepsilon} \mathcal{X}_{\omega_{\varepsilon}} u^{\varepsilon} \rightarrow u_{\mid \Gamma}^{0} \quad \text { in } C\left([0, T], H^{-1}(\Omega)\right) \text { if } \varepsilon \rightarrow 0
$$

and

$$
\lim _{\varepsilon \rightarrow 0} \frac{1}{\varepsilon} \int_{0}^{T} \int_{\omega_{\varepsilon}}\left|u^{\varepsilon}\right|^{2}=\int_{0}^{T} \int_{\Gamma}\left|u^{0}\right|^{2} .
$$

Proof: We prove this result in several steps.

Step 1. First, since $u^{\varepsilon} \in L^{\infty}\left((0, T), H^{1}(\Omega)\right)$ is bounded, by taking subsequences if necessary, we can assume that it converges weak ${ }^{*}$ in $L^{\infty}\left((0, T), H^{1}(\Omega)\right)$ to $u^{0}$; that is

$$
\left\langle u^{\varepsilon}, \varphi\right\rangle \rightarrow\left\langle u^{0}, \varphi\right\rangle \quad \text { as } \varepsilon \rightarrow 0 \quad \forall \varphi \in L^{1}\left((0, T), H^{-1}(\Omega)\right) .
$$

Step 2. From (3.18) and (3.6), with $s=1, q=r=2$, we have

$$
\frac{1}{\varepsilon} \int_{0}^{T} \int_{\omega_{\varepsilon}}\left|u^{\varepsilon}\right|^{2} \leq C \int_{0}^{T}\left\|u^{\varepsilon}\right\|_{H^{1}(\Omega)}^{2} \leq C .
$$

This and (3.19) implies, using Part B) in Lemma 3.3 (with $q=2=r$ ), that $W^{\varepsilon}=\frac{1}{\varepsilon} \mathcal{X}_{\omega_{\varepsilon}} u^{\varepsilon}$ is uniformly bounded in $H^{1}\left((0, T), H^{-1}(\Omega)\right) \subset C\left([0, T], H^{-1}(\Omega)\right)$.

Therefore, by taking subsequences again, if necessary, we can assume that

$$
W^{\varepsilon} \rightarrow W^{0} \quad \text { weakly in } H^{1}\left((0, T), H^{-1}(\Omega)\right) .
$$

At the same time from Part B) in Lemma 3.3 (with $q=2=r$ ), we get that

$$
W^{0} \in H^{1}\left((0, T), L^{2}(\Gamma)\right) \text {. }
$$

Step 3. We will prove that now $W^{0}=u_{\mid \Gamma}^{0}$ and then we get (3.20) and (3.21).

For this, consider $\varphi \in L^{2}\left((0, T), H^{1}(\Omega)\right)$ and then (3.15) gives

$$
\varphi_{\varepsilon}=\frac{1}{\varepsilon} \mathcal{X}_{\omega_{\varepsilon}} \varphi \rightarrow \varphi_{0}=\varphi_{\mid \Gamma} \quad \text { in } L^{1}\left((0, T), H^{-1}(\Omega)\right) \quad \text { as } \varepsilon \rightarrow 0
$$

and then from Step 1

$$
\left\langle u^{\varepsilon}, \varphi_{\varepsilon}\right\rangle=\frac{1}{\varepsilon} \int_{0}^{T} \int_{\omega_{\varepsilon}} u^{\varepsilon} \varphi=\left\langle W^{\varepsilon}, \varphi\right\rangle .
$$

Then the left hand side converges to $\left\langle u^{0}, \varphi_{0}\right\rangle=\int_{0}^{T} \int_{\Gamma} u^{0} \varphi$ while the right hand side converges to $\left\langle W^{0}, \varphi\right\rangle$. Hence, $W^{0}=u_{\mid \Gamma}^{0}$ as claimed.

Step 4. Now we prove (3.22) and for this we use Ascoli-Arzela's Theorem. First, we note that $W_{t}^{\varepsilon}$ is uniformly bounded in $L^{2}\left((0, T), H^{-1}(\Omega)\right)$ and then $W^{\varepsilon}(t, \cdot)$ is equicontinuous in $H^{-1}(\Omega), t \in(0, T)$. Second, we will prove that $W^{\varepsilon}(t, \cdot)$ is uniformly bounded in $H^{-s}(\Omega)$ for some $s<1$. Since $H^{-s}(\Omega) \subset H^{-1}(\Omega)$ is compact, we conclude the proof.

For this, take $r>2$ such that $H^{1}(\Omega) \subset L^{r}(\Gamma)$ and $s<1$ such that $H^{s}(\Omega) \subset$ $L^{r^{\prime}}(\Gamma)$, i.e. $-\frac{N-1}{r^{\prime}}<s-\frac{N}{2}<1-\frac{N}{2}$. Then by part A) in Lemma 3.1

$$
\left|\frac{1}{\varepsilon} \int_{\omega_{\varepsilon}} u^{\varepsilon} \varphi\right| \leq\left[\frac{1}{\varepsilon} \int_{\omega_{\varepsilon}}\left|u^{\varepsilon}\right|^{r}\right]^{\frac{1}{r}}\left[\frac{1}{\varepsilon} \int_{\omega_{\varepsilon}}|\varphi|^{r^{\prime}}\right]^{\frac{1}{r^{\prime}}} \leq C\left\|u^{\varepsilon}\right\|_{H^{1}(\Omega)}\|\varphi\|_{H^{s}(\Omega)} \leq C\|\varphi\|_{H^{s}(\Omega)} .
$$

That is, $\left\|W^{\varepsilon}(t, \cdot)\right\|_{H^{-s}(\Omega)} \leq C$ and we conclude. 
The last property in the statement follows from the weak convergence of $u^{\varepsilon}$ and the strong convergence of $\frac{1}{\varepsilon} \mathcal{X}_{\omega_{\varepsilon}} u^{\varepsilon} \cdot \square$

We will finally make use of the following result.

Lemma 3.8. Assume the family of potentials $V_{\varepsilon}$ is as in Lemma 3.5. Also, assume $u^{\varepsilon}$ is as in Lemma 3.7, that is, satisfies (3.18) and (3.19), and let $u^{0}$ be as in the conclusion of Lemma 3.7.

Then if $s$ is such that $\frac{1}{2}+\frac{N-1}{\rho}<s$, we have

$$
\frac{1}{\varepsilon} \mathcal{X}_{\omega_{\varepsilon}} V_{\varepsilon} u^{\varepsilon} \rightarrow V u_{\mid \Gamma}^{0} \quad \text { in } C\left([0, T], H^{-s}(\Omega)\right) .
$$

If, additionally,

$$
\rho>2(N-1)
$$

then

$$
\frac{1}{\varepsilon} \int_{0}^{T} \int_{\omega_{\varepsilon}} V_{\varepsilon}\left|u^{\varepsilon}\right|^{2} \rightarrow \int_{0}^{T} \int_{\Gamma} V\left|u^{0}\right|^{2} .
$$

Proof To prove (3.23) we use Ascoli-Arzela's Theorem as in Lemma 3.7 For this, denote $W^{\varepsilon}=\frac{1}{\varepsilon} \mathcal{X}_{\omega_{\varepsilon}} V_{\varepsilon} u^{\varepsilon}$. First, since as in Lemma 3.5] we have $\rho>N-1$, then for any $\frac{1}{2}+\frac{N-1}{2 \rho}<s^{*} \leq 1$ we have $H^{s^{*}}(\Omega) \subset L^{2 \rho^{\prime}}(\Gamma)$ and using (3.1) in Lemma 3.1

$$
\begin{aligned}
& \left|\left\langle\frac{1}{\varepsilon} \mathcal{X}_{\omega_{\varepsilon}} V_{\varepsilon} u^{\varepsilon}, \varphi\right\rangle\right| \leq\left[\frac{1}{\varepsilon} \int_{\omega_{\varepsilon}}\left|V_{\varepsilon}\right|^{\rho}\right]^{\frac{1}{\rho}}\left[\frac{1}{\varepsilon} \int_{\omega_{\varepsilon}}\left|u^{\varepsilon}\right|^{2 \rho^{\prime}}\right]^{\frac{1}{2 \rho^{\prime}}}\left[\frac{1}{\varepsilon} \int_{\omega_{\varepsilon}}|\varphi|^{2 \rho^{\prime}}\right]^{\frac{1}{2 \rho^{\prime}}} \\
& \leq C\left\|u^{\varepsilon}\right\|_{H^{s^{*}}(\Omega)}\|\varphi\|_{H^{s^{*}}(\Omega)} .
\end{aligned}
$$

Therefore, from (3.18), $W^{\varepsilon}$ is uniformly bounded in $L^{\infty}\left((0, T), H^{-s^{*}}(\Omega)\right)$.

Now observe that from (3.19) we have that $W_{t}^{\varepsilon}=\frac{1}{\varepsilon} \mathcal{X}_{\omega_{\varepsilon}} V_{\varepsilon} u_{t}^{\varepsilon}$ satisfies

$$
\begin{aligned}
& \left|\left\langle\frac{1}{\varepsilon} \mathcal{X}_{\omega_{\varepsilon}} V_{\varepsilon} u_{t}^{\varepsilon}, \varphi\right\rangle\right| \leq\left[\frac{1}{\varepsilon} \int_{\omega_{\varepsilon}}\left|V_{\varepsilon}\right|^{\rho}\right]^{\frac{1}{\rho}}\left[\frac{1}{\varepsilon} \int_{\omega_{\varepsilon}}\left|u_{t}^{\varepsilon}\right|^{2}\right]^{\frac{1}{2}}\left[\frac{1}{\varepsilon} \int_{\omega_{\varepsilon}}|\varphi|^{r}\right]^{\frac{1}{r}} \\
& \leq C\left[\frac{1}{\varepsilon} \int_{\omega_{\varepsilon}}\left|u_{t}^{\varepsilon}\right|^{2}\right]^{\frac{1}{2}}\left[\frac{1}{\varepsilon} \int_{\omega_{\varepsilon}}|\varphi|^{r}\right]^{\frac{1}{r}}
\end{aligned}
$$

with $\frac{1}{\rho}+\frac{1}{2}+\frac{1}{r}=1$ i.e. $r=\frac{2 \rho}{\rho-2}$.

Now, for any $s$ such that $\frac{1}{2}+\frac{N-1}{\rho}<s$ we have that $H^{s}(\Omega) \subset L^{r}(\Gamma)$, with $r=\frac{2 \rho}{\rho-2}$, and then have that, integrating in time in the inequality above and using Hölder's inequality

$$
\begin{aligned}
& \left|\int_{0}^{T}\left\langle\frac{1}{\varepsilon} \mathcal{X}_{\omega_{\varepsilon}} V_{\varepsilon} u_{t}^{\varepsilon}, \varphi\right\rangle\right| \leq C\left[\frac{1}{\varepsilon} \int_{0}^{T} \int_{\omega_{\varepsilon}}\left|u_{t}^{\varepsilon}\right|^{2}\right]^{\frac{1}{2}}\left[\int_{0}^{T}\left(\frac{1}{\varepsilon} \int_{\omega_{\varepsilon}}|\varphi|^{r}\right)^{\frac{2}{r}}\right]^{\frac{1}{2}} \\
& \leq C\left[\int_{0}^{T}\|\varphi\|_{H^{s}(\Omega)}^{2}\right]^{\frac{1}{2}}
\end{aligned}
$$

where we have used 3.19) and 3.1 in Lemma 3.1.

Hence, for $s>\frac{1}{2}+\frac{N-1}{\rho}, W_{t}^{\varepsilon}=\frac{1}{\varepsilon} \mathcal{X}_{\omega_{\varepsilon}} V_{\varepsilon} u_{t}^{\varepsilon}$ is uniformly bounded in

$$
L^{2}\left((0, T), H^{-s}(\Omega)\right) \text {. }
$$

In particular, $W^{\varepsilon}$ is uniformly bounded in $H^{1}\left((0, T), H^{-s}(\Omega)\right) \subset C\left([0, T], H^{-s}(\Omega)\right)$ and $W^{\varepsilon}$ is equicontinuous with values in $H^{-s}(\Omega)$. 
Now if $s$ satisfies $\frac{1}{2}+\frac{N-1}{\rho}<s$, then there exists $s^{*}$ satisfying $\frac{1}{2}+\frac{N-1}{2 \rho}<s^{*}<$ $\frac{1}{2}+\frac{N-1}{\rho}<s$. Then from the first part of the proof above we have that $W^{\varepsilon}(t, \cdot)$ is uniformly bounded in $H^{-s^{*}}(\Omega)$. Since $H^{-s^{*}}(\Omega) \subset H^{-s}(\Omega)$ is compact, we conclude the proof.

If we additionally assume (3.24), then we can take above $\frac{1}{2}+\frac{N-1}{\rho}<s \leq 1$. Then since, by Lemma 3.7 we have $u^{\varepsilon} \rightarrow u^{0}$, w- ${ }^{*}$ in $L^{\infty}\left((0, T), H^{1}(\Omega)\right)$, we get from (3.23),

$$
\frac{1}{\varepsilon} \int_{0}^{T} \int_{\omega_{\varepsilon}} V_{\varepsilon}\left|u^{\varepsilon}\right|^{2}=\left\langle\frac{1}{\varepsilon} \int_{0}^{T} \int_{\omega_{\varepsilon}} V_{\varepsilon} u^{\varepsilon}, u^{\varepsilon}\right\rangle \rightarrow\left\langle V u_{\mid \Gamma}^{0}, u^{0}\right\rangle=\int_{0}^{T} \int_{\Gamma} V\left|u^{0}\right|^{2}
$$

and we obtain (3.25).

\section{Singular limit as $\varepsilon \rightarrow 0$}

We analyze the limit of the solutions of the parabolic problems (1.1), with $0 \leq \varepsilon \leq \varepsilon_{0}$. For this we will assume that the data of the problem satisfy, for each $\varepsilon>0$ the assumptions in the first part of Theorem 2.2 with $h_{\varepsilon}=f_{\varepsilon}+\frac{1}{\varepsilon} \mathcal{X}_{\omega_{\varepsilon}} g_{\varepsilon}$ and the following uniform bounds in $\varepsilon>0$ :

$$
\begin{gathered}
\frac{1}{\varepsilon} \int_{\omega_{\varepsilon}}\left|V_{\varepsilon}\right|^{\rho} \leq C, \quad \text { some } \rho>N-1, \\
u_{0}^{\varepsilon} \in H^{1}(\Omega) \quad \text { and } \frac{1}{\varepsilon} \int_{\omega_{\varepsilon}}\left|u_{0}^{\varepsilon}\right|^{2} \leq C \\
f_{\varepsilon} \in L^{2}\left((0, T), L^{2}(\Omega)\right), \text { and } \int_{0}^{T}\left\|f_{\varepsilon}\right\|_{L^{2}(\Omega)}^{2} \leq C
\end{gathered}
$$

and

$$
\frac{1}{\varepsilon} \int_{0}^{T} \int_{\omega_{\varepsilon}}\left|g_{\varepsilon}\right|^{2} \leq C
$$

for some constant $C$ independent of $\varepsilon$.

Observe that in Theorem 2.2 we require $\lambda>-\lambda_{\Omega \backslash \overline{\omega_{\varepsilon}}}$ and now $\lambda_{\Omega \backslash \overline{\omega_{\varepsilon}}}>\lambda_{\Omega}$ and $\lambda_{\Omega \backslash \bar{\omega}_{\varepsilon}} \rightarrow \lambda_{\Omega}$ as $\varepsilon \rightarrow 0$. Thus, if $\lambda>-\lambda_{\Omega}$, then for sufficently small $\varepsilon$ we have $\lambda>-\lambda_{\Omega \backslash \bar{\omega}_{\varepsilon}}$. Hence we will also assume hereafter that

$$
\lambda>-\lambda_{\Omega} .
$$

Then, by Lemma 3.1 and 3.3. by taking subsequences if necessary, we can assume that there exists functions $V \in L^{\rho}(\Gamma), v_{0} \in L^{2}(\Gamma), f \in L^{2}\left((0, T), L^{2}(\Omega)\right)$ and $g \in L^{2}\left((0, T), L^{2}(\Gamma)\right)$ such that, as $\varepsilon \rightarrow 0$

$$
\begin{gathered}
\frac{1}{\varepsilon} \mathcal{X}_{\omega_{\varepsilon}} V_{\varepsilon} \rightarrow V \text { weakly in } H^{-s}(\Omega) \text { with } s-\frac{N}{2}>-\frac{N-1}{\rho^{\prime}}, \\
\frac{1}{\varepsilon} \mathcal{X}_{\omega_{\varepsilon}} u_{0}^{\varepsilon} \rightarrow v_{0} \text { weakly in } H^{-s}(\Omega) \text { with } s>\frac{1}{2} \\
\quad f_{\varepsilon} \rightarrow f \text { weakly in } L^{2}\left((0, T), L^{2}(\Omega)\right) \\
\frac{1}{\varepsilon} \mathcal{X}_{\omega_{\varepsilon}} g_{\varepsilon} \rightarrow g \text { weakly in } L^{2}\left((0, T), H^{-s}(\Omega)\right) \text { with } s>\frac{1}{2} .
\end{gathered}
$$


Also, observe that by [4.5], using the first part of Proposition 2.5 the problem (1.2) with initial data $v_{0} \in L^{2}(\Gamma)$, potential $V \in L^{\rho}(\Gamma)$ and nonhomogeneous terms $f \in L^{2}\left((0, T), L^{2}(\Omega)\right)$ and $g \in L^{2}\left((0, T), L^{2}(\Gamma)\right)$ is well posed.

Now observe that our proofs below rely on a compactness method based on suitable uniform estimates on solutions of (1.1). However assumption (4.5) alone may not be enough to guarantee that the elliptic operators in (1.1) and (1.2) are uniformly coercive in $\varepsilon$. Such uniform coercitivity is very helpful for estimates on solutions and can be achieved for $\lambda>\lambda_{0}$ for some $\lambda_{0}$ independent of $\varepsilon$; see part iii) in Lemma 3.5 This can be however overcame in a standad way by the usual change of variable $v^{\varepsilon}(t)=e^{\alpha t} u^{\varepsilon}(t)$ in (1.1), with a suitable $\alpha \in \mathbb{R}$. In fact the equation for $v^{\varepsilon}$ is similar to (1.1) but with the added term $\frac{\alpha}{\varepsilon} \mathcal{X}_{\omega_{\varepsilon}} v$. Hence using part i) in Lemma 3.5 $\alpha$ can be chosen, independent of $\varepsilon$ and in a suitable way such that uniform coerciveness follows. Observe that this approach requires modifying $f_{\varepsilon}$ and $g_{\varepsilon}$ but the assumptions (4.3), (4.4) and (4.8), (4.9) would still be satisfied by the new nonhomogeneous terms.

Note again that we make no assumption whatsoever on the signs of the concentrating or limit potentials, $V_{\varepsilon}, V$ nor we assume they are bounded (not even for fixed $\varepsilon$ ).

Then we have

THEOREM 4.1. Under the above notation, assume (4.6), 4.7), 4.8) and (4.9) and consider $u^{\varepsilon}$ the solutions of (1.1) as in the first part of Theorem 2.2. Moreover assume $\lambda_{0}>-\lambda_{\Omega}$. Also, let $u^{0}$ be the solution of (1.2) as in the first part of Proposition [2.5 with initial data $v_{0} \in L^{2}(\Gamma)$, potential $V \in L^{\rho}(\Gamma)$ and nonhomogeneous terms $f \in L^{2}\left((0, T), L^{2}(\Omega)\right)$ and $g \in L^{2}\left((0, T), L^{2}(\Gamma)\right)$.

Then, as $\varepsilon \rightarrow 0$,

$$
u^{\varepsilon} \rightarrow u^{0} \text { weakly in } L^{2}\left((0, T), H^{1}(\Omega)\right)
$$

and

$$
\begin{aligned}
\frac{1}{\varepsilon} \mathcal{X}_{\omega_{\varepsilon}} u^{\varepsilon} & \rightarrow u_{\mid \Gamma}^{0} \text { in } L^{2}\left((0, T), H^{-1}(\Omega)\right) \text { weakly, } \\
\frac{1}{\varepsilon} \mathcal{X}_{\omega_{\varepsilon}} V_{\varepsilon} u^{\varepsilon} & \rightarrow V u_{\mid \Gamma}^{0} \text { in } L^{2}\left((0, T), H^{-1}(\Omega)\right) \text { weakly. }
\end{aligned}
$$

In particular, for any $\varphi \in L^{2}\left((0, T), H^{1}(\Omega)\right)$

$$
\begin{gathered}
\frac{1}{\varepsilon} \int_{0}^{T} \int_{\omega_{\varepsilon}} u^{\varepsilon} \varphi \rightarrow \int_{0}^{T} \int_{\Gamma} u^{0} \varphi, \\
\frac{1}{\varepsilon} \int_{0}^{T} \int_{\omega_{\varepsilon}} V_{\varepsilon} u^{\varepsilon} \varphi \rightarrow \int_{0}^{T} \int_{\Gamma} V u^{0} \varphi .
\end{gathered}
$$

Proof We proceed in several steps. Observe that, as mentioned above, we can assume without loss of generality that the elliptic operators in (1.1) and (1.2) are uniformly coercive in $\varepsilon$. This happens for example if $\lambda>\lambda_{0}$ as in part iii) in Lemma 3.5

Step 1. Uniform bounds for $u^{\varepsilon}$.

Multiplying the equation by $u^{\varepsilon}$ in $L^{2}(\Omega)$, we get

$$
\begin{aligned}
& \frac{1}{2} \frac{d}{d t}\left(\frac{1}{\varepsilon} \int_{\omega_{\varepsilon}}\left|u^{\varepsilon}\right|^{2}\right)+\int_{\Omega}\left|\nabla u^{\varepsilon}\right|^{2}+\lambda \int_{\Omega}\left|u^{\varepsilon}\right|^{2} \\
& +\frac{1}{\varepsilon} \int_{\omega_{\varepsilon}} V_{\varepsilon}\left|u^{\varepsilon}\right|^{2}=\int_{\Omega} f_{\varepsilon} u^{\varepsilon}+\frac{1}{\varepsilon} \int_{\omega_{\varepsilon}} g_{\varepsilon} u^{\varepsilon} .
\end{aligned}
$$


Now from the uniform coercitivity, see e,g, iii) in Lemma 3.5

$$
C\left\|u^{\varepsilon}(t)\right\|_{H^{1}(\Omega)}^{2} \leq \int_{\Omega}\left|\nabla u^{\varepsilon}\right|^{2}+\lambda \int_{\Omega}\left|u^{\varepsilon}\right|^{2}+\frac{1}{\varepsilon} \int_{\omega_{\varepsilon}} V_{\varepsilon}\left|u^{\varepsilon}\right|^{2}
$$

for some $C>0$ independent of $\varepsilon$.

Next, applying Young's inequality, we obtain, for any $\delta>0$,

$$
\begin{gathered}
\left|\int_{\Omega} f_{\varepsilon} u^{\varepsilon}\right| \leq\left\|u^{\varepsilon}\right\|_{L^{2}(\Omega)}\left\|f_{\varepsilon}\right\|_{L^{2}(\Omega)} \leq \delta\left\|u^{\varepsilon}\right\|_{H^{1}(\Omega)}^{2}+\frac{1}{4 \delta}\left\|f_{\varepsilon}\right\|_{L^{2}(\Omega)}^{2} \\
\left|\frac{1}{\varepsilon} \int_{\omega_{\varepsilon}} g_{\varepsilon} u^{\varepsilon}\right| \leq\left(\frac{1}{\varepsilon} \int_{\omega_{\varepsilon}}\left|g_{\varepsilon}\right|^{2}\right)^{\frac{1}{2}}\left(\frac{1}{\varepsilon} \int_{\omega_{\varepsilon}}\left|u^{\varepsilon}\right|^{2}\right)^{\frac{1}{2}} \leq C\left\|u^{\varepsilon}\right\|_{H^{1}(\Omega)}\left(\frac{1}{\varepsilon} \int_{\omega_{\varepsilon}}\left|g_{\varepsilon}\right|^{2}\right)^{\frac{1}{2}} \leq \\
\leq \delta\left\|u^{\varepsilon}\right\|_{H^{1}(\Omega)}^{2}+\frac{C}{4 \delta} \frac{1}{\varepsilon} \int_{\omega_{\varepsilon}}\left|g_{\varepsilon}\right|^{2} .
\end{gathered}
$$

Now, taking $\delta$ enough small and integrating (4.10) in $t \in(0, T)$ and using (4.2), (4.3), (4.4), we obtain that for $0 \leq t \leq T$,

$$
\begin{aligned}
& \frac{1}{\varepsilon} \int_{\omega_{\varepsilon}}\left|u^{\varepsilon}(t)\right|^{2}+C \int_{0}^{t}\left\|u^{\varepsilon}\right\|_{H^{1}(\Omega)}^{2} \\
& \leq \frac{1}{\varepsilon} \int_{\omega_{\varepsilon}}\left|u_{0}^{\varepsilon}\right|^{2}+\frac{1}{2 \delta} \int_{0}^{t}\left\|f_{\varepsilon}\right\|_{L^{2}(\Omega)}^{2}+\frac{C}{2 \delta} \frac{1}{\varepsilon} \int_{0}^{t} \int_{\omega_{\varepsilon}}\left|g_{\varepsilon}\right|^{2} d t \leq C .
\end{aligned}
$$

Then, we have that

$$
\int_{0}^{T}\left\|u^{\varepsilon}(t)\right\|_{H^{1}(\Omega)}^{2} d t \leq C \quad \text { and } \quad \sup _{t \in[0, T]} \frac{1}{\varepsilon} \int_{\omega_{\varepsilon}}\left|u^{\varepsilon}(t)\right|^{2} \leq C .
$$

Step 2. Passing to the limit.

From (4.11) and Lemma 3.3 part B) with $q=r=2$, by taking subsequences if necessary, there exists a subsequence which converges weakly to $u^{0}$ in

$$
L^{2}\left((0, T), H^{1}(\Omega)\right)
$$

and there exists $w \in L^{2}\left((0, T), L^{2}(\Gamma)\right)=L^{2}((0, T) \times \Gamma)$ such that

$$
\frac{1}{\varepsilon} \mathcal{X}_{\omega_{\varepsilon}} u^{\varepsilon} \rightarrow w \quad \text { in } L^{2}\left((0, T), H^{-1}(\Omega)\right) \text { weakly as } \varepsilon \rightarrow 0 .
$$

Now, we prove that $w=u_{\mid \Gamma}^{0}$. For this, note that for every $\varphi \in L^{2}\left((0, T), H^{1}(\Omega)\right)$

$$
\left\langle\frac{1}{\varepsilon} \mathcal{X}_{\omega_{\varepsilon}} u^{\varepsilon}, \varphi\right\rangle=\frac{1}{\varepsilon} \int_{0}^{T} \int_{\omega_{\varepsilon}} u^{\varepsilon} \varphi=\left\langle u^{\varepsilon}, \frac{1}{\varepsilon} \mathcal{X}_{\omega_{\varepsilon}} \varphi\right\rangle
$$

Then, using (3.15) and taking another subsequence, if necessary, we obtain that

$$
\langle w, \varphi\rangle=\lim _{\varepsilon \rightarrow 0}\left\langle\frac{1}{\varepsilon} \mathcal{X}_{\omega_{\varepsilon}} u^{\varepsilon}, \varphi\right\rangle=\lim _{\varepsilon \rightarrow 0}\left\langle u^{\varepsilon}, \frac{1}{\varepsilon} \mathcal{X}_{\omega_{\varepsilon}} \varphi\right\rangle=\left\langle u^{0}, \varphi_{\mid \Gamma}\right\rangle=\int_{0}^{T} \int_{\Gamma} u^{0} \varphi .
$$

Thus $w=u_{\mid \Gamma}^{0}$.

Step 3. Next, we prove that $u^{0}$ satisfies the problem with dynamic boundary conditions (1.2) as in Proposition 2.5 
In order to get it, multiplying the equation from (1.1) by any smooth function $\varphi(t, x)$ we obtain

$$
\begin{aligned}
& \left\langle\frac{1}{\varepsilon} \mathcal{X}_{\omega_{\varepsilon}} u_{t}^{\varepsilon}, \varphi\right\rangle+\int_{0}^{T} \int_{\Omega} \nabla u^{\varepsilon} \nabla \varphi+\lambda \int_{0}^{T} \int_{\Omega} u^{\varepsilon} \varphi \\
& +\frac{1}{\varepsilon} \int_{0}^{T} \int_{\omega_{\varepsilon}} V_{\varepsilon} u^{\varepsilon} \varphi=\int_{0}^{T} \int_{\Omega} f_{\varepsilon} \varphi+\frac{1}{\varepsilon} \int_{0}^{T} \int_{\omega_{\varepsilon}} g_{\varepsilon} \varphi
\end{aligned}
$$

Now, assume $\varphi(T)=0$. Using Fubbini Theorem and integrating by parts, we rewrite the term $\left\langle\frac{1}{\varepsilon} \mathcal{X}_{\omega_{\varepsilon}} u_{t}^{\varepsilon}, \varphi\right\rangle$ to get

$$
\begin{gathered}
-\frac{1}{\varepsilon} \int_{0}^{T} \int_{\omega_{\varepsilon}} u^{\varepsilon} \varphi_{t}-\frac{1}{\varepsilon} \int_{\omega_{\varepsilon}} u^{\varepsilon}(0, \cdot) \varphi(0, \cdot)+\int_{0}^{T} \int_{\Omega} \nabla u^{\varepsilon} \nabla \varphi+\lambda \int_{0}^{T} \int_{\Omega} u^{\varepsilon} \varphi+ \\
\quad+\frac{1}{\varepsilon} \int_{0}^{T} \int_{\omega_{\varepsilon}} V_{\varepsilon} u^{\varepsilon} \varphi=\int_{0}^{T} \int_{\Omega} f_{\varepsilon} \varphi+\frac{1}{\varepsilon} \int_{0}^{T} \int_{\omega_{\varepsilon}} g_{\varepsilon} \varphi .
\end{gathered}
$$

Next, using (4.12) where $w=u_{\mid \Gamma}^{0}$ and applying (3.10) from Lemma 3.3 part B) with $q=r=2$, we have, as $\varepsilon \rightarrow 0$,

$$
\frac{1}{\varepsilon} \int_{0}^{T} \int_{\omega_{\varepsilon}} u^{\varepsilon} \varphi_{t} \rightarrow \int_{0}^{T} \int_{\Gamma} u^{0} \varphi_{t}, \quad \frac{1}{\varepsilon} \int_{0}^{T} \int_{\omega_{\varepsilon}} g_{\varepsilon} \varphi \rightarrow \int_{0}^{T} \int_{\Gamma} g \varphi
$$

and

$$
\frac{1}{\varepsilon} \int_{0}^{T} \int_{\omega_{\varepsilon}} V_{\varepsilon} u^{\varepsilon} \varphi \rightarrow \int_{0}^{T} \int_{\Gamma} V u^{0} \varphi
$$

Thus, taking the limit as $\varepsilon$ goes to zero in (4.13), we get

$$
\begin{gathered}
-\int_{\Gamma} v_{0} \varphi(0, \cdot)-\int_{0}^{T} \int_{\Gamma} u^{0} \varphi_{t}+\int_{0}^{T}\left[\int_{\Omega} \nabla u^{0} \nabla \varphi+\lambda \int_{\Omega} u^{0} \varphi\right] \\
+\int_{0}^{T} \int_{\Gamma} V u^{0} \varphi=\int_{0}^{T} \int_{\Omega} f \varphi+\int_{0}^{T} \int_{\Gamma} g \varphi .
\end{gathered}
$$

Thus, $u^{0}$ is a solution of (1.2) in the distributional sense, see Remark 2.6] and hence $u^{0}$ is the solution in the first part of Proposition 2.5] see Remark 2.7

Step 4. Now we show that all the family $u^{\varepsilon}$ converges and not only a subsequence.

In fact from the uniqueness for (1.2) in Remark 2.7 we have that from any subsequence in $u^{\varepsilon}$ there is another subsequence that converges to the same $u^{0}$. Hence the claim.

Now we impose stronger assumptions than (4.1) -4.4 on the data and obtain stronger convergence of solutions than in Theorem 4.1

More precisely, we assume now the initial conditions satisfy

$$
\left\|u_{0}^{\varepsilon}\right\|_{H^{1}(\Omega)}^{2} \leq C,
$$

and also the compatibility conditions on the initial data, (2.8) with $h=f_{\varepsilon}+\frac{1}{\varepsilon} \mathcal{X}_{\varepsilon} g_{\varepsilon}$, i.e.

$$
-\Delta u_{0}^{\varepsilon}+\lambda u_{0}^{\varepsilon}=f_{\varepsilon}(0) \text { in } \Omega \backslash \bar{\omega}_{\varepsilon} .
$$

Recall that $\lambda>-\lambda_{\Omega}$, see (4.5), and $\lambda_{\Omega \backslash \bar{\omega}_{\varepsilon}}>\lambda_{\Omega}$ and $\lambda_{\Omega \backslash \bar{\omega}_{\varepsilon}} \rightarrow \lambda_{\Omega}$ as $\varepsilon \rightarrow 0$. So for sufficently small $\varepsilon$ we have $\lambda>-\lambda_{\Omega \backslash \overline{\omega_{\varepsilon}}}$. 
We also assume

$$
f_{\varepsilon} \in H^{1}\left((0, T), L^{2}(\Omega)\right), \text { and }\left\|f_{\varepsilon}\right\|_{H^{1}\left((0, T), L^{2}(\Omega)\right)} \leq C
$$

and (4.4), where $C$ is a positive constant independent of $\varepsilon$.

Hence using (3.11) in Lemma 3.1] we have that $\frac{1}{\varepsilon} \int_{\omega_{\varepsilon}}\left|u_{0}^{\varepsilon}\right|^{2} \leq C\left\|u_{0}^{\varepsilon}\right\|_{H^{1}(\Omega)}^{2}$ and therefore (4.14) and (4.16) imply (4.2), (4.3) respectively.

Then by taking subsequences if necessary, we can assume (4.6), (4.7), (4.8) and (4.9). Moreover from Corollary 3.2 we have that in this case

$$
u_{0}^{\varepsilon} \rightarrow u_{0}^{0} \text { weakly in } H^{1}(\Omega) \text { and }\left.\frac{1}{\varepsilon} \mathcal{X}_{\varepsilon} u_{0}^{\varepsilon} \rightarrow u_{0}^{0}\right|_{\Gamma} \text { weakly in } H^{-1}(\Omega) .
$$

In particular $v_{0}=\left.u_{0}^{0}\right|_{\Gamma}$ in (4.7). Also, in (4.8) we have $f \in H^{1}\left((0, T), L^{2}(\Omega)\right)$ and

$$
f_{\varepsilon} \rightarrow f \text { weakly in } H^{1}\left((0, T), L^{2}(\Omega)\right) .
$$

Then we first make the following remark.

LEMMA 4.2. Under the above assumptions, we have

$$
-\Delta u_{0}^{0}+\lambda u_{0}^{0}=f(0) \quad \text { in } \Omega .
$$

Proof We first show that

$$
f_{\varepsilon}(0) \rightarrow f(0) \text { in } H^{-s}(\Omega), \quad 0<s<1
$$

and for this we use Ascoli-Arzela's Theorem. Observe that from 4.16 we have that $\left(f_{\varepsilon}\right)_{t}$ is uniformly bounded in $L^{2}\left((0, T), H^{-s}(\Omega)\right)$ for $0<s<1$ and then $f_{\varepsilon}$ is equicontinuous in $H^{-s}(\Omega), t \in(0, T)$. Second, from (4.16), we have that $f_{\varepsilon} \in H^{1}\left((0, T), L^{2}(\Omega)\right) \subset C\left([0, T], L^{2}(\Omega)\right)$ and therefore

$$
\sup _{0 \leq t \leq T}\left\|f_{\varepsilon}(t)\right\|_{L^{2}(\Omega)} \leq C .
$$

Hence $f_{\varepsilon}(t, \cdot)$ is uniformly bounded in $L^{2}(\Omega)$.

Finally, since $L^{2}(\Omega) \subset H^{-s}(\Omega)$ is compact, we conclude that $f_{\varepsilon} \rightarrow f$ in $C\left([0, T], H^{-s}(\Omega)\right)$, and the convergence of $f_{\varepsilon}(0)$ follows.

Now to prove (4.19) we consider $\varphi \in \mathcal{D}(\Omega)$ and small enough $\varepsilon$ such that $\operatorname{supp}(\varphi) \subset \Omega \backslash \omega_{\varepsilon}$. Thus, from (4.15) we have

$$
\int_{\Omega} \nabla u_{0}^{\varepsilon} \nabla \varphi+\lambda \int_{\Omega} u_{0}^{\varepsilon} \varphi=\int_{\Omega} f_{\varepsilon}(0) \varphi
$$

and taking the limit $\varepsilon \rightarrow 0$, using $u_{0}^{\varepsilon} \rightarrow u_{0}^{0}$ weakly in $H^{1}(\Omega)$ and the convergence of $f_{\varepsilon}(0)$, we obtain that

$$
\int_{\Omega} \nabla u_{0}^{0} \nabla \varphi+\lambda \int_{\Omega} u_{0}^{0} \varphi=\int_{\Omega} f(0) \varphi
$$

and we conclude.

Therefore, for each $\varepsilon>0$ we are under the assumptions in the second part of Theorem 2.2 with $h_{\varepsilon}=f_{\varepsilon}+\frac{1}{\varepsilon} \mathcal{X}_{\omega_{\varepsilon}} g_{\varepsilon}$. Also, for the limit problem (1.2) we are under the assumptions of the second part of Proposition 2.5 with initial data $v_{0}=\left.u_{0}^{0}\right|_{\Gamma} \in$ $L^{2}(\Gamma)$, potential $V \in L^{\rho}(\Gamma)$ and nonhomogeneous terms $f \in C\left([0, T], L^{2}(\Omega)\right)$ and $g \in L^{2}\left((0, T), L^{2}(\Gamma)\right)$. 4.1.

Hence, we have the following result that improves the convergence in Theorem 
THEOREM 4.3. Under the above notation, assume (4.4), 4.14, 4.15) and (4.16). Moreover assume $\lambda>-\lambda_{\Omega}$.

By taking subsequences if necessary, we can assume that the data satisfies (4.6), (4.7), 4.8) and (4.9) and moreover 4.17), 4.18) and (4.19).

Then if $u^{\varepsilon}$ and $u^{0}$ are as in Theorem 4.1. we have that in addition to the convergence in Theorem 4.1 we have now that $u^{\varepsilon}$ converges to $u^{0}$, weak ${ }^{*}$ in

$$
L^{\infty}\left((0, T), H^{1}(\Omega)\right)
$$

and

$$
\frac{1}{\varepsilon} \mathcal{X}_{\omega_{\varepsilon}} u^{\varepsilon} \rightarrow u_{\mid \Gamma}^{0} \in H^{1}\left((0, T), L^{2}(\Gamma)\right)
$$

weakly in $H^{1}\left((0, T), H^{-1}(\Omega)\right)$ and strongly in $C\left([0, T], H^{-1}(\Omega)\right)$. Also

$$
\frac{1}{\varepsilon} \mathcal{X}_{\omega_{\varepsilon}} V_{\varepsilon} u^{\varepsilon} \rightarrow V u_{\mid \Gamma}^{0} \quad \text { in } C\left([0, T], H^{-s}(\Omega)\right)
$$

for $\frac{1}{2}+\frac{N-1}{\rho}<s$.

If additionally $\rho>2(N-1)$ then $u^{\varepsilon}$ converges to $u^{0}$ also in $L^{2}\left((0, T), H^{1}(\Omega)\right)$.

Proof We proceed in several steps. Observe that, as mentioned above, we can assume without loss of generality that the elliptic operators in (1.1) and (1.2) are uniformly coercive in $\varepsilon$. This happens for example if $\lambda>\lambda_{0}$ as in part ii) in Lemma 3.5

Step 1. Uniform bounds on $u^{\varepsilon}$.

We note that we are under the assumptions of Theorem 2.2 and Proposition 2.3 and from (2.10) with $h=f_{\varepsilon}+\frac{1}{\varepsilon} \mathcal{X}_{\omega_{\varepsilon}} g_{\varepsilon}$, we have

$$
\begin{aligned}
& \frac{2}{\varepsilon} \int_{0}^{t} \int_{\omega_{\varepsilon}}\left|u_{t}^{\varepsilon}\right|^{2}+\int_{\Omega}\left|\nabla u^{\varepsilon}\right|^{2}+\lambda \int_{\Omega}\left|u^{\varepsilon}\right|^{2}+\frac{1}{\varepsilon} \int_{\omega_{\varepsilon}} V_{\varepsilon}\left|u^{\varepsilon}\right|^{2}= \\
= & \int_{\Omega}\left|\nabla u_{0}^{\varepsilon}\right|^{2}+\lambda \int_{\Omega}\left|u_{0}^{\varepsilon}\right|^{2}+\frac{1}{\varepsilon} \int_{\omega_{\varepsilon}} V_{\varepsilon}\left|u_{0}^{\varepsilon}\right|^{2}+\frac{2}{\varepsilon} \int_{0}^{t} \int_{\omega_{\varepsilon}} g_{\varepsilon} u_{t}^{\varepsilon}+ \\
+ & 2\left(\int_{0}^{t} \int_{\omega_{\varepsilon}} f_{\varepsilon} u_{t}^{\varepsilon}+\int_{\Omega \backslash \bar{\omega}_{\varepsilon}} f_{\varepsilon}(t) u^{\varepsilon}(t)-\int_{\Omega \backslash \bar{\omega}_{\varepsilon}} f_{\varepsilon}(0) u_{0}^{\varepsilon}-\int_{0}^{t} \int_{\Omega \backslash \bar{\omega}_{\varepsilon}}\left(f_{\varepsilon}\right)_{t} u^{\varepsilon}\right)
\end{aligned}
$$

Now, $\left(f_{\varepsilon}\right)_{t} \in L^{2}\left((0, T), L^{2}(\Omega)\right)$ and integrating by parts we obtain

$$
\int_{0}^{t} \int_{\omega_{\varepsilon}} f_{\varepsilon} u_{t}^{\varepsilon}=-\int_{0}^{t} \int_{\omega_{\varepsilon}}\left(f_{\varepsilon}\right)_{t} u^{\varepsilon}+\int_{\omega_{\varepsilon}} f_{\varepsilon}(t) u^{\varepsilon}(t)-\int_{\omega_{\varepsilon}} f_{\varepsilon}(0) u_{0}^{\varepsilon} .
$$

Hence, using the uniform corecitivity, see e.g. part iii) in Lemma 3.5 from (4.20) we have

$$
\begin{gathered}
\frac{2}{\varepsilon} \int_{0}^{t} \int_{\omega_{\varepsilon}}\left|u_{t}^{\varepsilon}\right|^{2}+C\left\|u^{\varepsilon}(t)\right\|_{H^{1}(\Omega)}^{2} \leq C\left\|u_{0}^{\varepsilon}\right\|_{H^{1}(\Omega)}^{2}+\frac{2}{\varepsilon} \int_{0}^{t} \int_{\omega_{\varepsilon}} g_{\varepsilon} u_{t}^{\varepsilon}+ \\
+2\left(\int_{\Omega} f_{\varepsilon}(t) u^{\varepsilon}(t)-\int_{\Omega} f_{\varepsilon}(0) u_{0}^{\varepsilon}-\int_{0}^{t} \int_{\Omega}\left(f_{\varepsilon}\right)_{t} u^{\varepsilon}\right) .
\end{gathered}
$$

Next, applying Young's inequality we get that

$$
\left|\frac{1}{\varepsilon} \int_{0}^{t} \int_{\omega_{\varepsilon}} g_{\varepsilon} u_{t}^{\varepsilon}\right| \leq \frac{1}{\delta \varepsilon} \int_{0}^{t} \int_{\omega_{\varepsilon}}\left|g_{\varepsilon}\right|^{2}+\delta \frac{1}{\varepsilon} \int_{0}^{t} \int_{\omega_{\varepsilon}}\left|u_{t}^{\varepsilon}\right|^{2}
$$


for any $\delta>0$. Using now

$$
\begin{aligned}
& \left|\int_{\Omega} f_{\varepsilon}(t) u^{\varepsilon}(t)-\int_{\Omega} f_{\varepsilon}(0) u_{0}^{\varepsilon}\right| \leq\left\|f_{\varepsilon}(t)\right\|_{L^{2}(\Omega)}\left\|u^{\varepsilon}(t)\right\|_{L^{2}(\Omega)} \\
& +\left\|u_{0}^{\varepsilon}\right\|_{L^{2}(\Omega)}\left\|f_{\varepsilon}(0)\right\|_{L^{2}(\Omega)}
\end{aligned}
$$

and applying again the Young inequality we get

$$
\begin{aligned}
& \left|\int_{\Omega} f_{\varepsilon}(t) u^{\varepsilon}(t)-\int_{\Omega} f_{\varepsilon}(0) u_{0}^{\varepsilon}\right| \leq \delta\left\|u^{\varepsilon}(t)\right\|_{H^{1}(\Omega)}^{2}+ \\
& \frac{1}{\delta}\left\|f_{\varepsilon}(t)\right\|_{L^{2}(\Omega)}^{2}+\delta\left\|u_{0}^{\varepsilon}\right\|_{H^{1}(\Omega)}^{2}+\frac{1}{\delta}\left\|f_{\varepsilon}(0)\right\|_{L^{2}(\Omega)}^{2}
\end{aligned}
$$

and proceeding as above

$$
\left|\int_{0}^{t} \int_{\Omega}\left(f_{\varepsilon}\right)_{t} u^{\varepsilon}\right| \leq \delta \int_{0}^{t}\left\|u^{\varepsilon}\right\|_{H^{1}(\Omega)}^{2}+\frac{1}{\delta} \int_{0}^{t}\left\|\left(f_{\varepsilon}\right)_{t}\right\|_{L^{2}(\Omega)}^{2} .
$$

Using these inequalities, from (4.21) we have that

$$
\begin{aligned}
& \frac{2(1-\delta)}{\varepsilon} \int_{0}^{t} \int_{\omega_{\varepsilon}}\left|u_{t}^{\varepsilon}\right|^{2}+(C-2 \delta)\left\|u^{\varepsilon}(t)\right\|_{H^{1}(\Omega)}^{2} \\
& \leq(C+2 \delta)\left\|u_{0}^{\varepsilon}\right\|_{H^{1}(\Omega)}^{2}+\frac{2}{\delta}\left\|f_{\varepsilon}(t)\right\|_{L^{2}(\Omega)}^{2}+ \\
& +\frac{2}{\delta}\left\|f_{\varepsilon}(0)\right\|_{L^{2}(\Omega)}^{2}+2 \delta \int_{0}^{t}\left\|u^{\varepsilon}\right\|_{H^{1}(\Omega)}^{2}+\frac{2}{\delta} \int_{0}^{t}\left\|\left(f_{\varepsilon}\right)_{t}\right\|_{L^{2}(\Omega)}^{2} \\
& +\frac{2}{\delta \varepsilon} \int_{0}^{t} \int_{\omega_{\varepsilon}}\left|g_{\varepsilon}\right|^{2} .
\end{aligned}
$$

Now from (4.16), and denoting $y(T)=\sup _{0 \leq t \leq T}\left\|u^{\varepsilon}(t)\right\|_{H^{1}(\Omega)}^{2}$ we get

$$
\delta \int_{0}^{t}\left\|u^{\varepsilon}\right\|_{H^{1}(\Omega)}^{2}+\frac{1}{\delta} \int_{0}^{t}\left\|\left(f_{\varepsilon}\right)_{t}\right\|_{L^{2}(\Omega)}^{2} \leq T \delta y(T)+\frac{1}{\delta} C .
$$

Also from (4.16), we have that $f_{\varepsilon} \in H^{1}\left((0, T), L^{2}(\Omega)\right) \subset C\left([0, T], L^{2}(\Omega)\right)$ and therefore

$$
\sup _{0 \leq t \leq T}\left\|f_{\varepsilon}(t)\right\|_{L^{2}(\Omega)} \leq C .
$$

Thus, from (4.22) and using also 4.4 we obtain

$$
\frac{2(1-\delta)}{\varepsilon} \int_{0}^{T} \int_{\omega_{\varepsilon}}\left|u_{t}^{\varepsilon}\right|^{2}+[C-2 \delta(1+T)] y(T) \leq C .
$$

Finally, taking $\delta<\min \left\{1, \frac{C}{2(1+T)}\right\}$ we conclude that

$$
\sup _{0 \leq t \leq T}\left\|u^{\varepsilon}(t)\right\|_{H^{1}(\Omega)}^{2} \leq C, \quad \text { and } \quad \frac{1}{\varepsilon} \int_{0}^{T} \int_{\omega_{\varepsilon}}\left|u_{t}^{\varepsilon}\right|^{2} \leq C .
$$

Step 2. Passing to the limit.

First, note that we are under the assumptions of Theorem 4.1 Hence $u^{\varepsilon}$ converges to some $u^{0}$ as in Theorem 4.1 But since the limit problem is as in the second part of Proposition 2.5 we have that extra regularity for $u^{0}$.

Next, from (4.23) we can apply Lemma [3.7 and then we have that $u^{\varepsilon}$ also converges to $u^{0}$ weak $k^{*}$ in $L^{\infty}\left((0, T), H^{1}(\Omega)\right)$ and

$$
\frac{1}{\varepsilon} \mathcal{X}_{\omega_{\varepsilon}} u^{\varepsilon} \rightarrow u_{\mid \Gamma}^{0} \quad \text { in } H^{1}\left((0, T), H^{-1}(\Omega)\right) \subset C\left([0, T], H^{-1}(\Omega)\right) .
$$


Also, from Lemma 3.8 for $\frac{1}{2}+\frac{N-1}{\rho}<s$, we get (3.23), i.e.

$$
\frac{1}{\varepsilon} \mathcal{X}_{\omega_{\varepsilon}} V_{\varepsilon} u^{\varepsilon} \rightarrow V u_{\mid \Gamma}^{0} \quad \text { in } C\left([0, T], H^{-s}(\Omega)\right) .
$$

Step 3. To conclude we prove the convergence in $L^{2}\left((0, T), H^{1}(\Omega)\right)$ provided that $\rho>2(N-1)$. For this, since we have weak convergence it is enough to prove convergence of the norm, that is, $\left\|u^{\varepsilon}\right\|_{L^{2}\left((0, T), H^{1}(\Omega)\right)} \rightarrow\left\|u^{0}\right\|_{L^{2}\left((0, T), H^{1}(\Omega)\right)}$ as $\varepsilon \rightarrow 0$.

Integrating in $t \in(0, T)$ the expression (4.10), we obtain that

$$
\begin{aligned}
& \frac{1}{2 \varepsilon} \int_{\omega_{\varepsilon}}\left|u^{\varepsilon}(T)\right|^{2}+\int_{0}^{T} E\left(u^{\varepsilon}(s)\right) d s+\frac{1}{\varepsilon} \int_{0}^{T} \int_{\omega_{\varepsilon}} V_{\varepsilon}\left|u^{\varepsilon}\right|^{2} \\
& =\frac{1}{2 \varepsilon} \int_{\omega_{\varepsilon}}\left|u_{0}^{\varepsilon}\right|^{2}+\int_{0}^{T} \int_{\Omega} f_{\varepsilon} u^{\varepsilon}+\frac{1}{\varepsilon} \int_{0}^{T} \int_{\omega_{\varepsilon}} g_{\varepsilon} u^{\varepsilon}
\end{aligned}
$$

where $E\left(u^{\varepsilon}\right)=\int_{\Omega}\left|\nabla u^{\varepsilon}\right|^{2}+\lambda \int_{\Omega}\left|u^{\varepsilon}\right|^{2}$.

Now observe that from Corollary 3.2 we have

$$
\frac{1}{\varepsilon} \int_{\omega_{\varepsilon}}\left|u_{0}^{\varepsilon}\right|^{2} \rightarrow \int_{\Gamma}\left|u_{0}\right|^{2}
$$

while

$$
\frac{1}{\varepsilon} \int_{\omega_{\varepsilon}}\left|u^{\varepsilon}(T)\right|^{2} \rightarrow \int_{\Gamma}\left|u^{0}(T)\right|^{2} .
$$

For this last statement, observe that, from (4.23), $\left\|u^{\varepsilon}(T)\right\|_{H^{1}(\Omega)}^{2} \leq C$, and from the convergence in Step 2, he have

$$
\frac{1}{\varepsilon} \mathcal{X}_{\omega_{\varepsilon}} u^{\varepsilon}(T) \rightarrow u_{\mid \Gamma}^{0}(T) \quad \text { strongly in } H^{-1}(\Omega) .
$$

Hence, the arguments in Corollary 3.2 allow to conclude.

Next, assuming $\rho>2(N-1)$ from [3.25) in Lemma 3.8 we get

$$
\frac{1}{\varepsilon} \int_{0}^{T} \int_{\omega_{\varepsilon}} V_{\varepsilon}\left|u^{\varepsilon}\right|^{2} \rightarrow \int_{0}^{T} \int_{\Gamma} V\left|u^{0}\right|^{2}
$$

Therefore, passing to the limit in the energy equality above, we obtain that

$$
\begin{aligned}
& \frac{1}{2} \int_{\Gamma}\left|u^{0}(T)\right|^{2}+\lim _{\varepsilon \rightarrow 0}\left(\int_{0}^{T} E\left(u^{\varepsilon}(s)\right) d s\right)+\int_{0}^{T} \int_{\Gamma} V\left|u^{0}\right|^{2} \\
& =\frac{1}{2} \int_{\Gamma}\left|u_{0}\right|^{2}+\int_{0}^{T} \int_{\Omega} f u^{0}+\int_{0}^{T} \int_{\Gamma} g u^{0} .
\end{aligned}
$$

On the other hand, multiplying (1.2) by $u^{0}$ in $L^{2}(\Omega)$ and integrating by parts, we get

$$
\frac{1}{2} \frac{d}{d t} \int_{\Gamma}\left|u^{0}\right|^{2}+E\left(u^{0}\right)+\int_{\Gamma} V\left|u^{0}\right|^{2}=\int_{\Omega} f u^{0}+\int_{\Gamma} g u^{0}
$$

with $E\left(u^{0}\right)=\int_{\Omega}\left|\nabla u^{0}\right|^{2}+\lambda \int_{\Omega}\left|u^{0}\right|^{2}$. Integrating in $t \in(0, T)$ the expression above, we obtain that

$$
\begin{aligned}
& \frac{1}{2} \int_{\Gamma}\left|u^{0}(T)\right|^{2}+\int_{0}^{T} E\left(u^{0}\right)+\int_{0}^{T} \int_{\Gamma} V\left|u^{0}\right|^{2} \\
& =\frac{1}{2} \int_{\Gamma}\left|u_{0}\right|^{2}+\int_{0}^{T} \int_{\Omega} f u^{0}+\int_{0}^{T} \int_{\Gamma} g u^{0} .
\end{aligned}
$$


and comparing with (4.24) we conclude that

$$
\int_{0}^{T} E\left(u^{0}(s)\right) d s=\lim _{\varepsilon \rightarrow 0} \int_{0}^{T} E\left(u^{\varepsilon}(s)\right) d s
$$

and we get that $u^{\varepsilon}$ converges to $u^{0}$ in $L^{2}\left((0, T), H^{1}(\Omega)\right)$.

\section{References}

[1] D. Andreucci, P. Bisegna, E. DiBenedetto, "Homogenization and concentrated capacity for the heat equation with non-linear variational data in reticular almost disconnected structures and applications to visual transduction", Ann. Mat. Pura Appl. (4) 182, no. 4, 375-407 (2003)

[2] J.M. Arrieta, A. Jiménez-Casas, A.Rodríguez-Bernal, "Nonhomogeneous flux condition as limit of concentrated reactions ", Revista Iberoamericana de Matematicas, vol 24, no 1, 183211, (2008).

[3] C. Bardos, G. Lebeau, J. Rauch, Sharp sufficient conditions for the observation control and stabilization of waves from the boundary, SIAM J. Control Optim., 30, 10241065 (1992).

[4] C. Cavaterra, C. Gal, M. Grasselli, A. Miranville, "Phase-field systems with nonlinear coupling and dynamic boundary conditions", Nonlinear Anal., vol.72, 5, 2375-2399, (2010).

[5] J. Escher, "Nonlinear elliptic systems with dynamic boundary conditions", Math. Z., vol. 210 , 3, 413-439, (1992).

[6] J.Z. Farkas, P. Hinow, "Physiologically structured populations with diffusion and dynamic boundary conditions", Math. Biosci. Eng., vol. 8, 2, 503-513, (2011).

[7] C. Gal, M. Grasselli, "The non-isothermal Allen-Cahn equation with dynamic boundary conditions", Discrete Contin. Dyn. Syst., vol. 22 ,4, 1009-1040, (2008).

[8] G. Gilardi, A. Miranville, G. Schimperna, " On the Cahn-Hilliard equation with irregular potentials and dynamic boundary conditions", Commun. Pure Appl. Anal., vol. 8 , 3, 881-912, (2009).

[9] M. Grasselli, A. Miranville, G. Schimperna, The Caginalp phase-field system with coupled dynamic boundary conditions and singular potentials. Discrete Contin. Dyn. Syst. 28, no. 1, 67-98 (2010).

[10] M. Grobbelaar-van Dalsen, N. Sauer, "Solutions in Lebesgue spaces of the Navier-Stokes equations with dynamic boundary

conditions", Proc. Roy. Soc. Edinburgh Sect. A., vol. 123, 4,745-761, (1993).

[11] Á. Jiménez-Casas, A. Rodríguez-Bernal, "Singular limit for a nonlinear parabolic equation with terms concentrating on the boundary", J. Math. Anal. and Apl. 379, 567-588 (2011).

[12] V. Komornik, E. Zuazua, A direct method for the boundary stabilization of the wave equation, J. Math. Pures Appl., 69, 3355 (1990).

[13] J. Lagnese, Note on the boundary stabilization of wave equations, SIAM J. Control Optim., 26, 12501256 (1988).

[14] J. Lagnese, Boundary Stabilization of Thin Plates , SIAM Studies in Appl. Math., vol. 10, (1989).

[15] I. Lasiecka and R. Triggiani, Control Theory for Partial Differential Equations: Continuous and Approximation Theories, vol. 1 and 2, Cambridge University Press, (2000).

[16] J.L.Lions, Quelques Méthodes de Rèsolution des Problèmes aux Limites non Lineaires, Dunod (1969).

[17] A. Miranville, S.Zelik, "The Cahn-Hilliard equation with singular potentials and dynamic boundary conditions", Discrete Contin. Dyn. Syst., vol. 28 , 1, 275-310, (2010).

[18] A. Rodríguez-Bernal, E. Zuazua, "Parabolic Singular Limit of a Wave Equation with Localized Boundary Damping", Dis. Cont. Dyn. Sys., vol.1, 3, 303-346, (1995).

[19] A. Rodríguez-Bernal, E. Zuazua , "Parabolic Singular Limit of a Wave Equation with Localized Interior Damping", Comm. Contem. Math. , vol.3, 2, 215-257, (2001).

[20] G. Savaré, A. Visintin, "Variational convergence of nonlinear diffusion equations: applications to concentrated capacity problems with change of phase", Atti Accad. Naz. Lincei Cl. Sci. Fis. Mat. Natur. Rend. Lincei (9) Mat. Appl. 8, no. 1, 49-89 (1997).

[21] M. A. Storti, N.M. Nigro, R. Paz, L. Dalcin, "Dynamic boundary conditions in computational fluid dynamics", Comput. Methods Appl. Mech. Engrg., vol. 197, 13-16, 1219-1232, (2008). 
[22] A.Toyohiko , "Two-phase Stefan problems with dynamic boundary conditions", Adv. Math. Sci. Appl., vol. 2, 2, 253-270, (1993).

[23] Xiao, Ti-Jun; Liang, Jin Second order parabolic equations in Banach spaces with dynamic boundary conditions. Trans. Amer. Math. Soc. 356, no. 12, 47874809 (2004).

Grupo de Dinámica No Lineal, Universidad Pontificia Comillas de Madrid, C/Alberto Aguilera 23, 28015 MADrid, Spain

E-mail address: ajimenez@dmc.icai.upcomillas.es

Departamento de Matemática Aplicada, Universidad Complutense de Madrid, 28040 Madrid and Instituto de Ciencias Matemáticas, CSIC-UAM-UC3M-UCM, Spain 